\title{
Formal GNS Construction and States in Deformation Quantization
}

\author{
M. Bordemann*, S. Waldmann ${ }^{\S}$ \\ Fakultät für Physik \\ Universität Freiburg \\ Hermann-Herder-Str. 3 \\ 79104 Freiburg i. Br., F. R. G
}

FR-THEP-96/12

July 1996

\begin{abstract}
In this paper we develop a method of constructing Hilbert spaces and the representation of the formal algebra of quantum observables in deformation quantization which is an analog of the well-known GNS construction for complex $C^{*}$-algebras: in this approach the corresponding positive linear functionals ('states') take their values not in the field of complex numbers, but in (a suitable extension field of) the field of formal complex Laurent series in the formal parameter. By using the algebraic and topological properties of these fields we prove that this construction makes sense and show in physical examples that standard representations as the Bargmann and Schrödinger representation come out correctly, both formally and in a suitable convergence scheme. For certain Hamiltonian functions (contained in the Gel'fand ideal of the positive functional) a formal solution to the time-dependent Schrödinger equation is shown to exist. Moreover, we show that for every Kähler manifold equipped with the Fedosov star product of Wick type all the classical delta functionals are positive and give rise to some formal Bargmann representation of the deformed algebra.
\end{abstract}

*mbor@phyq1.physik.uni-freiburg.de

§waldman@phyq1.physik.uni-freiburg.de 


\section{Introduction}

In the programme of deformation quantization introduced by Bayen, Flato, Fronsdal, Lichnerowicz and Sternheimer [4] the algebra of quantum observables is considered as an associative local formal deformation (a so-called star product $*$ ) of the associative commutative algebra of smooth complex-valued functions $C^{\infty}(M)$ on a given symplectic manifold $M$, such that the first order commutator equals $i \lambda$ times the Poisson bracket and such that complex conjugation is an antilinear involution of the deformed algebra. The latter is equal to $C^{\infty}(M)[[\lambda]]$, the space of formal power series in the deformation parameter $\lambda$ with coefficients in $C^{\infty}(M)$, and the associative noncommutative multiplication $*$ is bilinear with respect to the ring $\mathbb{C}[[\lambda]]$ of formal power series in $\lambda$ with complex coefficients. A lot of progress has been made on the question of existence of these deformations for general symplectic manifolds (DeWilde-Lecomte 1983 [13]; Fedosov 1985 [15]; Omori-Maeda-Yoshioka 1991 [26]), and of existence of strongly invariant deformations under Lie groups preserving a torsion-free connection on $M$ (Fedosov 1996 [16, p.180]).

On the other hand star products have the -at first sight rather unpleasant- feature of lacking convergence uniform in the formal parameter which is due to the fact that they depend on the infinite jets of the two functions which in turn can be made as divergent as possible by Borel's Theorem (see e.g. [33]; see also the article by Rubio 1984 [29] for the commutativity of local associative products on $\left.C^{\infty}(M)\right)$. Moreover, the deformed algebras do not seem to have obvious representations in some complex separable Hilbert space which is unsatisfactory from the physical point of view. In the past decade, however, several people have attacked this problem: Cahen, Gutt, and Rawnsley [10], [11] start from the finite-dimensional operator algebras of geometric quantization in tensor powers of a very ample regular prequantum line bundle over a compact Kähler manifold and use coherent states (see [6], [28]) to first construct star products for the Berezin-Rawnsley symbols ( 6 ], [28]) for each tensor power separately. In a second step an asymptotic expansion of these star products in the inverse tensor power is shown to define a local star product on the manifold where the formal parameter appears as a sort of interpolation of the inverse tensor powers. For compact Hermitean symmetric spaces they showed that the subspace of representative functions has a convergent star product. See also [7], [8] for an elementary algebraic approach in the particular case of complex projective space. Pflaum has studied star products and their convergence on suitable subspaces on cotangent bundles in his thesis [27. Moreover, Fedosov has shown that the deformed algebra allows a so-called asymptotic operator representation in a complex Hilbert space if and only if certain integrality properties of a formal index are satisfied which he defines as a formal analogue of the Atiyah-Singer index theorem and which is an invariant of the underlying symplectic manifold (see e. g. Fedosov's book [16] for a detailed exposition).

The approach of this paper is motivated by the following consideration: in the theory of complex $C^{*}$-algebras which form one of the main mathematical pillars of algebraic quantum field theory (see Haag's book [20] for details) the representing complex Hilbert spaces for a given complex $C^{*}$-algebra $A$ are constructed by means of the so-called GNS representation (named after the mathematicians Gel'fand, Naimark, and Segal, see [5]): roughly speaking, any positive complex-valued linear functional on $A$ (i.e. which maps positive elements of $A$ to nonnegative real numbers) gives rise to a left ideal $I$ of $A$ (the Gel'fand ideal), and $A$ 
is canonically represented on the quotient space $A$ modulo $I$. Due to the positivity of the initial functional this quotient is equipped with a positive definite sesqulilinear form and thus becomes a complex pre-Hilbert space whose completion yields the desired representation of the algebra. The initial functional can be regarded as a vacuum expectation value (functional) or a state on $A$ if $A$ has a unit element.

A natural question which has been brought to our attention by K. Fredenhagen is the following: can the GNS construction be extended to the associative algebras occurring in deformation quantization? At first sight, this seems almost impossible: firstly, for any complex-valued linear functional on $C^{\infty}(M)[[\lambda]]$ one would at once have an obvious convergence problem. Secondly, although the deformed algebra does have an antilinear involution it does not seem to share the remaining defining analytic properties of a complex $C^{*}$-algebra such as the existence of a $C^{*}$-norm or any obvious uniform structure in which it is complete.

This picture, however, will change drastically -as we should like to show in the sequelwhen the linear functionals take their values not only in the field of complex numbers but can have values in (some suitable field extension of) the ring $\mathbb{C}[[\lambda]]$ and are not only linear with respect to $\mathbb{C}$, but $\mathbb{C}[[\lambda]]$-linear which makes sense since $C^{\infty}(M)[[\lambda]]$ is a $\mathbb{C}[[\lambda]]$-module in a natural way. Thereby one stays in the formal category which avoids the convergence problems. Moreover, in the subring $\mathbb{R}[[\lambda]]$ of formal power series with real coefficients there is an algebraic sense of 'asymptotic positivity': a power series in $\mathbb{R}[[\lambda]]$ is defined to be positive (resp. negative) if and only if among its nonvanishing coefficients the one with lowest order is a positive (resp. negative) real number. This defines a ring ordering (which is preserved under sums and products). This structure allows us to speak of a positive linear functional $\omega$ on the deformed algebra if and only if $\omega(\bar{f} * f)$ is nonnegative in $\mathbb{R}[[\lambda]]$ for all $f \in C^{\infty}(M)[[\lambda]]$. For technical reasons it will be advantageous to replace the ring $\mathbb{R}[[\lambda]]$ by its quotient field $\mathbb{R}((\lambda)$ ) of formal Laurent series (with finite principal part) and likewise for $\mathbb{C}[[\lambda]]$ and $C^{\infty}(M)[[\lambda]]$.

By this simple replacement of the complex numbers by (field extensions of) formal power series we get the following principal results:

- With the above notion of positive linear functionals with values in (a field extension of) $\mathbb{C}((\lambda))$ we can imitate the algebraic part of the classical GNS construction for the algebras in deformation quantization and arrive at a representation of these algebras in a pre-Hilbert space over (a suitable field extension) of $\mathbb{C}((\lambda))$ (section 3 ).

- The above 'asymptotic ordering' of the field defines a metric topology on the field which can be used to define a norm on the above pre-Hilbert space with values in (a suitable extension of $\mathbb{R}((\lambda))$. This norm serves to define a Cauchy completion of the pre-Hilbert space (section 3).

- Apart from this algebraic construction this 'formal' GNS construction gives rise to the geometric interpretation of these positive linear functionals as a deformation (quantization) of classical positive linear functionals: these classical 'states' in classical mechanics are often given by certain measures (e.g. the Boltzmann distribution) having support on certain submanifolds of $M$. In this paper we shall deal with the simplest examples: a) Dirac measures (whose support is one point in $M$ ) for which we shall show in the case of the standard Wick star product in $\mathbb{C}^{n}$ that the above formal GNS con- 
struction gives rise to the formal Wick quantization with the correct formal Bargmann Hilbert space (section 5), and b) the integral over configuration space $\mathbb{R}^{n}$ in $\mathbb{R}^{2 n}$ for the standard star product of Weyl type which will give rise to the usual symmetrization rule and the correct formal $L^{2}$-space of (certain) square-integrable functions of Schrödinger quantization on configuration space (section 7).

- Moreover, we show that for the Fedosov star product of Wick type on an arbitrary Kähler manifold (see [9] for details) the Dirac delta functionals are positive functionals on the deformed algebra which allows a representation of Bargmann type. The geometry of the Kähler manifold enters this representation by mapping the function $f \in C^{\infty}(M)((\lambda))$ on its Fedosov-Taylor series at the support of the delta functional (section 6).

- Finally, in all the above examples we could also find a solution to the problem of convergence of formal powers series: either by using a given formal Hilbert base (in the Kähler examples) or a set of suitable formal linear functionals (in the case of the formal Schrödinger representation) we define a complex Hilbert space out of the formal GNS Hilbert space for the value $\lambda=\hbar$ in the following way: we first single out the subset $H(\hbar)$ of all those elements $\psi$ of the formal Hilbert space which satisfy an infinite number of suitable convergence conditions for all those formal series with complex coefficients which arise when all the scalar products of $\psi$ with the elements of the Hilbert base or the values of all the given linear functionals on $\psi$ are considered. This turns out to be a complex vector space and the quotient space $\mathcal{H}(\hbar):=H(\hbar) / N(\hbar)$ becomes a complex Hilbert space where $N(\hbar)$ is the subspace of those elements of $H(\hbar)$ for which all the above series vanish when $\lambda$ is replaced by the real number $\hbar$. A certain complex subspace of the formal algebra will respect this construction and can thus be represented on (a subspace of) $\mathcal{H}(\hbar)$.

These examples seem to support the point of view that the convergence problem in deformation quantization should be treated only after a formal GNS representation has been chosen (by the choice of a suitable positive linear functional), i.e. one should "stay in the formal category as long as possible".

The paper is organized as follows: Section 2 recalls the necessary notation and definitions in deformation quantization, introduces the use of the field of formal Laurent series and gives some first elementary examples and counterexamples of formal positive functionals. The main flaw of the field of formal Laurent series is the fact that it is not algebraically closed. Section 3 is rather technical: here we first describe the properties of general ordered fields we shall need such as their algebraic extensions and closures, their topology and absolute values. We then define Hilbert spaces over (certain) ordered fields and finally describe the GNS construction of associative algebras with a *-involution over certain algebraic extensions of these fields. The ideas of the proofs of many of these results can be found in algebra text books, or in the literature on $p$-adic functional analysis (see e.g. [25]), or in the works on (nonclassical) Hilbert spaces (see e.g. [19], 24, [18]). In section 4 we apply the results of section 3 to the concrete fields we need for the GNS construction in deformation quantization, i.e. the algebraic closure of the Laurent field, the so-called field of Newton-Puiseux series, and its Cauchy completion. The GNS construction is applied to the formal algebra $C^{\infty}(M)\langle\langle\lambda\rangle\rangle$ 
of completed Newton-Puiseux series with coefficients in $C^{\infty}(M)$ which contains the original deformed algebra $C^{\infty}(M)[[\lambda]]$ as a subring. Moreover, we prove that a formal solution to the time-dependent Schrödinger equation exists as a well-defined curve of vectors in the formal GNS Hilbert space in case the classical Hamiltonian does not contain any negative powers of $\lambda$ and is contained in the Gel'fand ideal of the considered positive linear functional $\omega$. Section 5 contains the above-mentioned example of a GNS construction by means of the delta functional with support at the origin for the Wick star product in $\mathbb{C}^{n}$. In addition we obtain the correct spectrum of the harmonic oscillator by an entirely formal deduction. Section 6 generalizes this to the delta functional of an arbitrary point of an arbitrary Kähler manifold equiped with the Fedosov star product of Wick type. In section 7 the abovementioned example of the formal Schrödinger representation in $\mathbb{R}^{n}$ is dealt with. Section 8 is an outlook on some open problems related to this approach.

\section{Motivation and basic concepts}

In this section we shall give a first heuristic motivation how one can define positive linear functionals for a star product algebra without leaving the formal description. First we have to introduce some notation: Let $M$ be a $2 n$-dimensional symplectic manifold. The observable algebra in deformation quantization is given by the formal power series $C^{\infty}(M)[[\lambda]]$ in the formal parameter $\lambda$ with coefficients in the smooth complex-valued functions $C^{\infty}(M)$. Then the addition in $C^{\infty}(M)[[\lambda]]$ is the pointwise addition order by order in $\lambda$ of the functions and the multiplication is a star product for $M$. A star product of two functions $f, g \in C^{\infty}(M)$ is a formal power series

$$
f * g=\sum_{r=0}^{\infty} \lambda^{r} M_{r}(f, g)
$$

with bilinear operators $M_{r}: C^{\infty}(M) \times C^{\infty}(M) \rightarrow C^{\infty}(M)$ such that $*$ extends to a $\mathbb{C}[[\lambda]]$ bilinear associative product for $C^{\infty}(M)[[\lambda]]$ with the following properties: The lowest order is the pointwise multiplication, i.e. $M_{0}(f, g)=f g$, and the first order commutator is given by $i \lambda$ times the Poisson bracket, i. e. $M_{1}(f, g)-M_{1}(g, f)=i\{f, g\}$. The constant function $\mathbf{1}$ is the unit element with respect to $*$, i.e. $f * \mathbf{1}=\mathbf{1} * f=f$. We assume for simplicity that all the operators $M_{r}$ are not only local but bidifferential operators. We demand in addition that the complex conjugation of elements in $C^{\infty}(M)[[\lambda]]$ (where $\bar{\lambda}:=\lambda$ ) is an antilinear algebra involution:

$$
\overline{f * g}=\bar{g} * \bar{f}
$$

Then $C^{\infty}(M)[[\lambda]]$ together with a star product is not only a *-algebra over the field $\mathbb{C}$ but also a $\mathbb{C}[[\lambda]]$-module.

It is now an easy step to pass form the ring of formal power series to the corresponding quotient field which turns out to be the field of formal Laurent series. The field of formal Laurent series with real (and analogously complex) coefficients is defined by

$$
\mathbb{R}((\lambda)):=\left\{\sum_{r=-N}^{\infty} \lambda^{r} a_{r} \mid a_{r} \in \mathbb{R}, N \in \mathbb{Z}\right\} .
$$


Then $\mathbb{R}((\lambda))$ has the structure of a field if we define the addition order by order in $\lambda$ and the multiplication by

$$
\left(\sum_{r=-N}^{\infty} \lambda^{r} a_{r}\right)\left(\sum_{s=-M}^{\infty} \lambda^{s} b_{s}\right):=\sum_{t=-N-M}^{\infty} \lambda^{t} \sum_{\substack{r+s=t \\ r \geq-N, s \geq-M}} a_{r} b_{s} .
$$

Then one easily shows that $\mathbb{R}((\lambda))$ and $\mathbb{C}((\lambda))$ are fields and with $\bar{\lambda}:=\lambda$ we have the following natural inclusions: $\mathbb{R} \subset \mathbb{R}[[\lambda]] \subset \mathbb{R}((\lambda)), \mathbb{C} \subset \mathbb{C}[[\lambda]] \subset \mathbb{C}((\lambda))$ and $\mathbb{R}((\lambda)) \subset \mathbb{C}((\lambda))$. Moreover $\mathbb{R}((\lambda))$ is the quotient field of the ring $\mathbb{R}[[\lambda]]$ and now $\lambda$ has an inverse, namely $\lambda^{-1}$.

Remark: From the physical point of view it will be necessary to consider negative powers of Planck's constant anyway since $\hbar$ will appear in the denominator in important situations as for example in the energy eigenvalues of the hydrogen atom.

More generally, if $V$ is a $\mathbb{K}$-vector space $(\mathbb{K}=\mathbb{R}, \mathbb{C})$ then we define the corresponding vector space of formal Laurent series $V((\lambda))$ by

$$
V((\lambda)):=\left\{\sum_{r=-N}^{\infty} \lambda^{r} v_{r} \mid v_{r} \in V, N \in \mathbb{Z}\right\}
$$

and notice that $V((\lambda))$ is a $\mathbb{K}((\lambda))$-vector space in the obvious way. Moreover the field $\mathbb{R}((\lambda))$ is known to be an ordered field with an unique ordering (e. g. [31, p. 73]):

Lemma 2.1 $\mathbb{R}((\lambda))$ is an ordered field with an unique non-archimedian ordering relation such that $\lambda>0$. The positive elements are given by

$$
\sum_{r=-N}^{\infty} \lambda^{r} a_{r}>0 \Longleftrightarrow a_{-N}>0 .
$$

A symbolic picture of this non-archimedian ordering is given by

$$
\cdots<\lambda^{-1} \mathbb{R}^{-}<\mathbb{R}^{-}<\lambda \mathbb{R}^{-}<\cdots<0<\cdots<\lambda \mathbb{R}^{+}<\mathbb{R}^{+}<\lambda^{-1} \mathbb{R}^{+}<\cdots .
$$

Apart from this ordering relation the fields $\mathbb{R}((\lambda))$ and $\mathbb{C}((\lambda))$ allow an absolute value $\varphi$ : $\mathbb{C}((\lambda)) \rightarrow \mathbb{R}$ defined for $a \in \mathbb{C}((\lambda))$ by

$$
\varphi(a):=2^{-o(a)}
$$

where $o(a) \in \mathbb{Z}$ is the order of the lowest non-vanishing term in the formal Laurent series $a$. Both structures, the order and the absolute value, can be used to define topologies on the field $\mathbb{R}((\lambda)$ ) (see section 3 for definitions) which will turn out to be the same (see section 4 ). An important observation will be that in $\mathbb{R}((\lambda))$ every Cauchy sequence defined with respect to the order converges in $\mathbb{R}((\lambda))$.

Using the fields $\mathbb{R}((\lambda))$ and $\mathbb{C}((\lambda))$ instead of $\mathbb{R}$ and $\mathbb{C}$ we can generalize the $\mathbb{C}$-algebra of observables $C^{\infty}(M)[[\lambda]]$ in the obvious way to a $\mathbb{C}((\lambda))$-algebra $C^{\infty}(M)((\lambda))$ where we extend the star product to a $\mathbb{C}((\lambda))$-bilinear product of $C^{\infty}(M)((\lambda))$. Now we can consider $\mathbb{C}((\lambda))$-linear functionals $\omega: C^{\infty}(M)((\lambda)) \rightarrow \mathbb{C}((\lambda))$ and define positive linear functionals using the complex conjugation as antilinear algebra involution and the ordering of the $\mathbb{R}((\lambda))$ completely analogously to the case of $C^{*}$-algebras over $\mathbb{C}[5]$ : 
Definition $2.2 A \mathbb{C}((\lambda))$-linear functional $\omega: C^{\infty}(M)((\lambda)) \rightarrow \mathbb{C}((\lambda))$ is called positive iff

$$
\omega(\bar{f} * f) \geq 0 \quad \forall f \in C^{\infty}(M)((\lambda))
$$

and a state iff $\omega$ is positive and $\omega(\mathbf{1})=1$.

We will now give simple examples for positive linear functionals. Using the locality of the star product we can prove the following lemma:

Lemma 2.3 Let $0 \leq \varrho \in C^{0}(M)$ with compact support. Then $\omega_{\varrho}$ defined by

$$
\omega_{\varrho}(f):=\int_{M} f \varrho \Omega \quad(\Omega \text { symplectic volume form })
$$

is a positive linear functional of $C^{\infty}(M)((\lambda))$ with respect to any star product and $\omega_{\varrho}(\bar{f} * f)=$ $0 \Longleftrightarrow f \varrho \equiv 0$.

A counterexample is given by the delta-functionals in $\mathbb{R}^{2}$ and the Weyl-Moyal star product (63). We easily compute that the evaluation functional $\delta_{\left(q_{0}, p_{0}\right)}$ at $\left(q_{0}, p_{0}\right) \in \mathbb{R}^{2}$ is not positive since

$$
\delta_{\left(q_{0}, p_{0}\right)}\left(\overline{\left(\left(q-q_{0}\right)^{2}+\left(p-p_{0}\right)^{2}\right)} *\left(\left(q-q_{0}\right)^{2}+\left(p-p_{0}\right)^{2}\right)\right)=-\frac{1}{2} \lambda^{2}<0
$$

in spite of the fact that the delta functional is a positive functional with respect to the pointwise product and hence a classical state.

Now it is possible to construct a representation of the $\mathbb{C}((\lambda))$-algebra $C^{\infty}(M)((\lambda))$ using a given positive linear functional in the same way as the usual GNS representation of a $C^{*}$ algebra is constructed. But this will be done in a more general context in the next section and then it will turn out that there is even a better choice than the formal Laurent series since the field $\mathbb{R}((\lambda))$ is not real closed and $\mathbb{C}((\lambda))$ is not algebraically closed since for example the positive element $\lambda$ is no square.

\section{Ordered fields and general GNS construction}

In this rather technical section we shall present some general properties of ordered fields and vector spaces and algebras over such fields. All statements can in principal be proved in a fashion very similar to the case of real or complex numbers, and for the proofs only the ordering axioms will be used. In order to make this exposition not too long we shall omit most of the proofs. As general references we have used the textbooks by Jacobson [21] (in particular Chapter 5) and [22] (Chapters 9, 11), by Ruiz [31], Kelley [23], Rudin [30], Yosida [34], Bratteli-Robinson [5], and Haag [20].

Definition 3.1 ([21, Def. 5.1]) An ordered field $(\mathrm{R}, P)$ is field $\mathrm{R}$ with a subset $P$ of positive elements of $\mathrm{R}$ such that $0 \notin P$ and if $a \in \mathrm{R}$ then either $a=0, a \in P$ or $-a \in P$ and if $a, b \in P$ then $a+b \in P$ and $a b \in P$. Then an ordering relation is defined by $a<b$ iff $b-a \in P$. 
The symbols $>, \leq$ and $\geq$ will be used as in the case $\mathrm{R}=\mathbb{R}$. The elements with $a<0$ will be called negative. The set of positive elements will also be denoted by $\mathrm{R}^{+}$and the negative elements by $\mathrm{R}^{-}$. Clearly, every square $a^{2}$ is positive or 0 and hence $-1<0<1$. Since $n 1=1+\cdots+1$ is a sum of positive numbers for all $n \in \mathbb{N}$ we have $n 1>0$ and hence $\mathrm{R}$ has characteristic zero. We define $|a|:=a$ if $a \geq 0$ and $|a|:=-a$ if $a<0$. Then $|\cdot|$ clearly satisfies

$$
\begin{gathered}
|a| \geq 0 \quad \text { and } \quad|a|=0 \Longleftrightarrow a=0 \\
\quad|a b|=|a||b| \\
|| a|-| b|| \leq|a+b| \leq|a|+|b| .
\end{gathered}
$$

The ordering relation $<$ is called archimedian iff there is for any $0<a$ and $b \in \mathrm{R}$ a natural number $n \in \mathbb{N}$ such that $n a>b$. Otherwise the ordering will be called non-archimedian.

Now we fix once and for all the quadratic field extension $C:=\mathrm{R}(i)(=\mathrm{R} \oplus i \mathrm{R})$ of an ordered field $\mathrm{R}$ where $i^{2}:=-1$. In $\mathrm{C}$ we define complex conjugation as usual by $a+i b \in$ $\mathrm{C} \mapsto \overline{a+i b}:=a-i b$ where $a, b \in \mathrm{R}$. Then the complex conjugation is an involutive field automorphism. The elements of the subfield $\mathrm{R}$ of $\mathrm{C}$ will be called real and are characterised as usual by $a \in \mathrm{R} \subset \mathrm{C} \Longleftrightarrow \bar{a}=a$. Clearly $\bar{a} a \in \mathrm{R}, \bar{a} a \geq 0$ and $\bar{a} a=0 \Longleftrightarrow a=0$ for any $a \in \mathrm{C}$ and we define $|a|^{2}:=\bar{a} a$.

Now we transfer definitions and some simple algebraic results from the theory of complex vector spaces with Hermitian products to the general case of a vector space over an arbitrary ordered field $\mathrm{R}$ and its quadratic field extension $\mathrm{C}$.

Definition 3.2 Let $\mathrm{C}=\mathrm{R}(i)$ be the quadratic field extension of an ordered field $\mathrm{R}$ and $\mathfrak{H}_{\mathrm{C}}$ a C-vector space. A map $\langle\cdot, \cdot\rangle: \mathfrak{H}_{\mathrm{C}} \times \mathfrak{H}_{\mathrm{C}} \rightarrow \mathrm{C}$ is called a Hermitian product iff for all $\phi, \psi, \chi \in \mathfrak{H}_{\mathrm{C}}$ and for all $a, b \in \mathrm{C}$

i.) $\langle\cdot, \cdot\rangle$ is antilinear in the first argument, i.e. $\langle a \phi+b \psi, \chi\rangle=\bar{a}\langle\phi, \chi\rangle+\bar{b}\langle\psi, \chi\rangle$

ii.) $\langle\phi, \psi\rangle=\overline{\langle\psi, \phi\rangle}$

iii.) $\langle\phi, \phi\rangle \geq 0$ and $\langle\phi, \phi\rangle=0 \Longleftrightarrow \phi=0$.

A C-vector space with a Hermitian product $\langle\cdot, \cdot\rangle$ is called a pre-Hilbert space. A linear map $U: \mathfrak{H}_{\mathrm{C}} \rightarrow \mathfrak{K}_{\mathrm{C}}$ from one pre-Hilbert space over $\mathrm{C}$ to another is called an isometry iff $\langle U \phi, U \psi\rangle=\langle\phi, \psi\rangle$ for all $\phi, \psi \in \mathfrak{H}_{\mathrm{C}}$ and unitary iff $U$ is a bijective isometry.

For a pre-Hilbert space we have the Cauchy-Schwarz inequality for the Hermitian product which is a simple imitation of the standard arguments over the complex numbers (cf e.g. [30, p. 77]):

Lemma 3.3 (Cauchy-Schwarz inequality) Let $\mathfrak{H}_{\mathrm{C}}$ be a pre-Hilbert space with Hermitian product $\langle\cdot, \cdot\rangle$ and $\phi, \psi \in \mathfrak{H}_{\mathrm{c}}$. Then

$$
\langle\phi, \psi\rangle\langle\psi, \phi\rangle \leq\langle\phi, \phi\rangle\langle\psi, \psi\rangle
$$

with equality if and only if $\psi$ and $\phi$ are linearly dependent.

Furthermore, for a linear operator from $\mathfrak{H}_{\mathbf{C}}$ into $\mathfrak{H}_{\mathbf{C}}$ we want to define an adjoint operator. In general it is rather complicated to see whether an adjoint operator exists or not. Without further assumptions we can only state the following definition for everywhere defined linear operators: 
Definition 3.4 (Adjoint operator) Let $\mathfrak{H}_{\mathrm{C}}$ be a pre-Hilbert space and $A: \mathfrak{H}_{\mathrm{C}} \rightarrow \mathfrak{H}_{\mathrm{C}}$ a linear map. Then a linear map $B: \mathfrak{H}_{\mathrm{C}} \rightarrow \mathfrak{H}_{\mathrm{C}}$ is called an adjoint operator to $A$ iff $\langle\phi, A \psi\rangle=$ $\langle B \phi, \psi\rangle$ for all $\phi, \psi \in \mathfrak{H}_{\mathrm{c}}$. In this case we write $B=A^{*}$.

Lemma 3.5 Let $\mathfrak{H}_{\mathrm{C}}$ be a pre-Hilbert space and $A, B: \mathfrak{H}_{\mathrm{C}} \rightarrow \mathfrak{H}_{\mathrm{C}}$ linear operators. If $A^{*}$ exists then it is unique and if $A^{*}$ and $B^{*}$ exist then $(a A+b B)^{*},\left(A^{*}\right)^{*}$ and $(A B)^{*}$ exist for $a, b \in \mathrm{C}$ and $(a A+b B)^{*}=\bar{a} A^{*}+\bar{b} B^{*},\left(A^{*}\right)^{*}=A$ and $(A B)^{*}=B^{*} A^{*}$.

Let $\mathcal{A}$ be an associative and not necessarily commutative algebra over the quadratic field extension $\mathrm{C}=\mathrm{R}(i)$ of an ordered field $\mathrm{R}$. We shall only consider algebras with an antilinear involution * compatible with the complex conjugation in $\mathrm{C}$. This means that there is a map * $: \mathcal{A} \rightarrow \mathcal{A}$ such that for all $A, B \in \mathcal{A}$ and $a \in \mathrm{C}$

$$
\begin{aligned}
(A+B)^{*} & =A^{*}+B^{*} & (a A)^{*} & =\bar{a} A^{*} \\
(A B)^{*} & =B^{*} A^{*} & \left(A^{*}\right)^{*} & =A .
\end{aligned}
$$

If the algebra $\mathcal{A}$ has a unit element 1 then necessarily $\mathbf{1}^{*}=\mathbf{1}$. By means of the algebra involution * and the ordering relation in $\mathrm{R}$ we can define positive linear functionals analogously to the case of $C^{*}$-algebras over the complex numbers $\mathbb{C}$ [5, 20]:

Definition 3.6 Let $\mathcal{A}$ be a $\mathrm{C}$-algebra with involution * and $\omega: \mathcal{A} \rightarrow \mathrm{C}$ a linear functional where $\mathrm{C}=\mathrm{R}(i)$ and $\mathrm{R}$ is an ordered field. Then $\omega$ is called positive iff for all $A \in \mathcal{A}$

$$
\omega\left(A^{*} A\right) \geq 0 .
$$

If in addition $\mathcal{A}$ has a unit element $\mathbf{1}$ then $\omega$ is called a state iff $\omega$ is positive and $\omega(\mathbf{1})=1$.

For a positive linear functional we can prove the Cauchy-Schwarz inequality:

Lemma 3.7 (Cauchy-Schwarz inequality) Let $\mathcal{A}$ be a $\mathrm{C}$-algebra with involution * and $\omega: \mathcal{A} \rightarrow \mathrm{C}$ a positive linear functional. Then we have for all $A, B \in \mathcal{A}$ :

$$
\begin{aligned}
\omega\left(A^{*} B\right) & =\overline{\omega\left(B^{*} A\right)} \\
\omega\left(A^{*} B\right) \overline{\omega\left(A^{*} B\right)} & \leq \omega\left(A^{*} A\right) \omega\left(B^{*} B\right)
\end{aligned}
$$

Moreover $\omega(\mathbf{1})=0$ implies $\omega \equiv 0$ and $\omega\left(A^{*}\right)=\overline{\omega(A)}$ and if $\omega\left(A^{*} A\right)=0$ then $\omega\left(A^{*} B\right)=0$ for all $B \in \mathcal{A}$.

For an algebra $\mathcal{A}$ with involution * over the quadratic field extension $\mathrm{C}$ of an ordered field R we are able to transfer the well-known GNS construction of representations for $C^{*}$ algebras: We construct a representation of $\mathcal{A}$ in a pre-Hilbert space over $\mathrm{C}$. Firstly, we shall only deal with the algebraic properties of this construction and examine analytic properties later. We are mainly using the notation of [5]. Let $\omega: \mathcal{A} \rightarrow \mathrm{C}$ be a positive linear functional. Then we consider the following subspace of $\mathcal{A}$ :

$$
\mathcal{J}_{\omega}:=\left\{A \in \mathcal{A} \mid \omega\left(A^{*} A\right)=0\right\}
$$


This subspace $\mathcal{J}_{\omega}$ is called the Gel'fand ideal of $\omega$ and by means of lemma 3.7 it is easily proved that $\mathcal{J}_{\omega}$ is indeed a left ideal of $\mathcal{A}$. In the next step one considers the quotient vector space

$$
\mathfrak{H}_{\omega}:=\mathcal{A} / \mathcal{J}_{\omega}
$$

where the equivalence classes in $\mathfrak{H}_{\omega}$ are denoted by

$$
\psi_{A}:=\left\{A^{\prime} \in \mathcal{A} \mid A^{\prime}=A+I, I \in \mathcal{J}_{\omega}\right\}
$$

On the quotient space $\mathfrak{H}_{\omega}$ we can define a Hermitian product by

$$
\left\langle\psi_{A}, \psi_{B}\right\rangle:=\omega\left(A^{*} B\right)
$$

which is well-defined since $\mathcal{J}_{\omega}$ is a left ideal. Furthermore this definition leads indeed to a non-degenerate Hermitian product for $\mathfrak{H}_{\omega}$ which will make $\mathfrak{H}_{\omega}$ a pre-Hilbert space over C. In a third step one defines a representation $\pi_{\omega}$ of $\mathcal{A}$ on $\mathfrak{H}_{\omega}$ by

$$
\pi_{\omega}(A) \psi_{B}:=\psi_{A B}
$$

To prove that this is well-defined we need once more the fact that $\mathcal{J}_{\omega}$ is a left ideal. Then we notice that $\pi_{\omega}$ is a ${ }^{*}$-representation: $\pi_{\omega}(A B)=\pi_{\omega}(A) \pi_{\omega}(B), \pi_{\omega}$ is linear and $\pi_{\omega}\left(A^{*}\right)=$ $\pi_{\omega}(A)^{*}$. In this case it is checked directly that $\pi_{\omega}\left(A^{*}\right)$ is the adjoint operator of $\pi_{\omega}(A)$ in the sense of definition 3.4. If $\mathcal{A}$ has an unit element 1 and $\omega$ is a state then the representation $\pi_{\omega}$ is cyclic with the cyclic (vacuum) vector $\psi_{\mathbf{1}}$ since every vector $\psi_{A} \in \mathfrak{H}_{\text {c }}$ can be written as $\psi_{A}=\pi_{\omega}(A) \psi_{\mathbf{1}}$ and we have

$$
\omega(A)=\left\langle\psi_{1}, \pi_{\omega}(A) \psi_{\mathbf{1}}\right\rangle
$$

In this case we show analogously to the case of $C^{*}$-algebras that the representation $\pi_{\omega}$ is unique up to unitary equivalence: If $\mathfrak{H}^{\prime}, \pi^{\prime}, \psi^{\prime}$ is another cyclic ${ }^{*}$-representation with cyclic unit vector $\psi^{\prime}$ such that $\omega(A)=\left\langle\psi^{\prime}, \pi^{\prime}(A) \psi^{\prime}\right\rangle$ for all $A \in \mathcal{A}$ then we have an unitary map $U: \mathfrak{H}_{\omega} \rightarrow \mathfrak{H}^{\prime}$ such that $U^{-1} \pi^{\prime}(A) U=\pi(A)$ and $U \psi_{\mathbf{1}}=\psi^{\prime}$. We shall resume this in the following theorem:

Theorem 3.8 (General GNS construction) Let $\mathcal{A}$ be a C-algebra with involution * where $\mathrm{C}=\mathrm{R}(i)$ is the quadratic field extension of an ordered field $\mathrm{R}$. For any positive linear functional there exists $a^{*}$-representation $\pi_{\omega}$ on a pre-Hilbert space $\mathfrak{H}_{\omega}$ as constructed above which is called the GNS representation. If $\mathcal{A}$ in addition has an unit element 1 and $\omega$ is a state then this representation is cyclic and we have $\omega(A)=\left\langle\psi_{\mathbf{1}}, \pi_{\omega}(A) \psi_{\mathbf{1}}\right\rangle$ and this property defines this representation up to unitary equivalence.

The following obvious generalization will be very useful if the positive linear functional is only defined on a proper ideal of $\mathcal{A}$, a situation which will occur in section 7 .

Corollary 3.9 Let $\mathcal{A}$ be a $\mathrm{C}$-algebra with involution * and $\mathcal{B} \subset \mathcal{A}$ a twosided ideal of $\mathcal{A}$ with $\mathcal{B}=\mathcal{B}^{*}$. Let $\omega: \mathcal{B} \rightarrow \mathcal{C}$ be a positive linear functional and denote by $\pi_{\omega}$ the GNS representation of $\mathcal{B}$ in $\mathfrak{H}_{\omega}=\mathcal{B} / \mathcal{J}_{\omega}$ where $\mathcal{J}_{\omega} \subset \mathcal{B}$ is the Gel'fand ideal of $\omega$. If $\mathcal{J}_{\omega}$ is a left ideal of $\mathcal{A}$ then $\pi_{\omega}$ could be extended canonically to a representation of $\mathcal{A}$ on $\mathfrak{H}_{\omega}$. 
Now we shall concentrate on some 'analytic' properties of ordered fields. Hence we shall examine some standard definitions of topology and calculus and transfer them from the case of real numbers $\mathbb{R}$ to the more general case of an arbitrary ordered field R. Again all the statements can be proved analogously using only the ordering axioms.

First of all we define $\epsilon$-balls around any point in $\mathrm{R}$ by means of the ordering relation and notice that the ordering induces a topology and a uniform structure for $\mathrm{R}$ similar to the topology and the uniform structure of a metric space [23, Chap. 6].

Definition 3.10 Let $\mathrm{R}$ be an ordered field. For any $x \in \mathrm{R}$ and any $0<\epsilon \in \mathrm{R}$ we define the $\epsilon$-ball around $x$ by

$$
B_{\epsilon}(x):=\{y \in \mathrm{R}|| x-y \mid<\epsilon\} .
$$

The set of all $\epsilon$-balls is denoted by $\mathcal{B}:=\left\{B_{\epsilon}(x) \subseteq \mathrm{R} \mid 0<\epsilon \in \mathrm{R}, x \in \mathrm{R}\right\}$. Furthermore we define for $0<\epsilon \in \mathrm{R}$

$$
U_{\epsilon}:=\{(x, y) \in \mathrm{R} \times \mathrm{R}|| x-y \mid<\epsilon\} \subseteq \mathrm{R} \times \mathrm{R}
$$

and $\mathcal{U}^{\prime}:=\left\{U_{\epsilon} \subseteq \mathrm{R} \times \mathrm{R} \mid 0<\epsilon \in \mathrm{R}\right\}$

Proposition 3.11 The set of $\epsilon$-balls $\mathcal{B}$ is a base of a topology $\mathcal{T}$ for the field $\mathrm{R}$ such that $\mathrm{R}$ becomes a normal space. The set $\mathcal{U}^{\prime}$ is a base for an uniform structure $\mathcal{U}$ for $\mathrm{R}$ which induces the same topology $\mathcal{T}$ than $\mathcal{B}$. Addition and multiplication are uniformly continuous maps from $\mathrm{R} \times \mathrm{R} \rightarrow \mathrm{R}$.

We shall call this topology and the uniform structure induced by the ordering relation the standard topology and the standard uniform structure of the ordered field R. Using this topology we can define continuous functions $f: \mathrm{R} \rightarrow \mathrm{R}$ as usual.

Remark: We notice that in the topology induced by the ordering relation the intervals may neither be connected nor compact in general. As an example we consider the field of formal Laurent series $\mathbb{R}((\lambda))$ over the real numbers and the open balls $B_{n \lambda}(r)$ with $r \in \mathbb{R}$ and $n \in \mathbb{N}$. Then for $a<b$ the closed interval $[a, b]:=\{x \mid a \leq x \leq b\} \subset \mathbb{R}((\lambda))$ in the Laurent field is contained in

$$
[a, b] \subset \bigcup_{\substack{n \in \mathbb{N} \\ r \in[a, b] \subset \mathbb{R}}} B_{n \lambda}(r)
$$

but $B_{n \lambda}(r) \cap B_{n^{\prime} \lambda}\left(r^{\prime}\right)=\emptyset$ if $r \neq r^{\prime}$. Hence $[a, b] \subset \mathbb{R}((\lambda))$ is neither compact nor connected.

Another important concept is the supremum and infimum of a bounded subset in an ordered field. We define them as usual: A subset $U \subseteq \mathrm{R}$ is called bounded iff there is a $C \in \mathrm{R}$ such that $|x| \leq C$ for all $x \in U$. Then $C$ is called a bound for $U$. Analogously we define upper and lower bounds. $C$ is called the supremum (infimum) of $U$ iff $C$ is the smallest upper bound (largest lower bound), i.e. for all $0<\epsilon \in \mathrm{R}$ there exists $x \in U$ such that $x>C-\epsilon(x<C+\epsilon)$. In an archimedian ordered field the existence of a supremum or infimum of a bounded set can be proved iff the field is Cauchy complete. On the other hand, in a non-archimedian ordered field neither the supremum nor the infimum of a bounded set exist in general [32, p. 245]. 
Definition 3.12 (Convergence, Cauchy sequences, completeness) Let $\mathrm{R}$ be an ordered field and $\left(a_{n}\right)_{n \in \mathbb{N}}$ a sequence in $\mathrm{R}$. Then $\left(a_{n}\right)$ is called convergent to a $\in \mathrm{R}$ iff

$$
\forall 0<\epsilon \in \mathrm{R} \quad \exists N \in \mathbb{N} \text { such that } \forall n>N:\left|a_{n}-a\right|<\epsilon .
$$

A sequence $\left(a_{n}\right)$ is called a Cauchy sequence iff

$$
\forall 0<\epsilon \in \mathrm{R} \quad \exists N \in \mathbb{N} \text { such that } \forall m, n>N:\left|a_{m}-a_{n}\right|<\epsilon .
$$

An ordered field is called Cauchy complete iff every Cauchy sequence converges in $\mathrm{R}$.

It is a well-known result that for any ordered field $R$ there exists an unique ordered field $\widehat{R}$ such that $\mathrm{R}$ is a dense subfield of $\widehat{R}$, the orderings are compatible and $\widehat{R}$ is Cauchy complete [32, p. 238].

For certain approximation statements we shall need to know whether the topology or the uniform structure is first countable or not (a topology for a set is called first countable iff any point has a countable base of neighbourhoods [23, p. 50]). In our case the existence of such a countable base of neigbourhoods is equivalent to the existence of non-trivial zero-sequences:

Proposition 3.13 Let $\mathrm{R}$ be an ordered field. Then the following properties are equivalent:

i.) There is a sequence $\left(\epsilon_{n}\right)_{n \in \mathbb{N}}$ such that $\epsilon_{n}>0$ for all $n \in \mathbb{N}$ and $\epsilon_{n} \rightarrow 0$.

ii.) The standard uniform structure $\mathcal{U}$ has a countable base.

iii.) The standard topology $\mathcal{T}$ is first countable.

iv.) The standard uniform structure $\mathcal{U}$ and the standard topology $\mathcal{T}$ can be induced by a metric.

Proof: The implications $i$.) $\Rightarrow$ ii.) $\Rightarrow$ iii.) are obvious and $i i i.) \Rightarrow i$.) is proved by constructing the zero sequence using a given countable neighbourhood base. The fourth part is equivalent to the second one for general reasons since $\mathcal{T}$ is Hausdorff according to proposition 3.11 (cf. e.g. [23, p. 186]).

Although the supremum of a bounded subset does not exist in general we can easily prove the existence of a sequence in a bounded subset with supremum which converges to the supremum if the field is first countable:

Lemma 3.14 Let $\mathrm{R}$ be an ordered field such that the standard topology is first countable. If for $A \subset \mathrm{R}$ the supremum $\sup A$ exists then there is a sequence $\left(a_{n}\right)$ with elements in $A$ such that $a_{n} \rightarrow \sup A$.

In the theory of fields another possibility to define a metric topology is an absolute value:

Definition 3.15 (Absolute value [21, p. 558]) Let $\mathrm{R}$ be a field. An absolute value $\varphi$ is a map $\varphi: \mathrm{R} \rightarrow \mathbb{R}^{+} \cup\{0\}$ such that for all $a, b \in \mathrm{R}$

i.) $\varphi(a)=0 \Longleftrightarrow a=0$ 


$$
\begin{aligned}
& \text { ii.) } \varphi(a b)=\varphi(a) \varphi(b) \\
& \text { iii.) } \varphi(a+b) \leq \varphi(a)+\varphi(b) \text {. }
\end{aligned}
$$

An absolute value $\varphi$ is called non-archimedian iff $\varphi(a+b) \leq \max (\varphi(a), \varphi(b))$ and archimedian if this is not the case. An absolute value is called trivial iff $\varphi(0)=0$ and $\varphi(a)=1$ for all $0 \neq a \in \mathrm{R}$.

With help of an absolute value one can define a metric on $\mathrm{R}$ for $a, b \in \mathrm{R}$ by

$$
d_{\varphi}(a, b):=\varphi(a-b) .
$$

Recall that if the absolute value is non-archimedian then the metric $d_{\varphi}$ is an ultrametric, i.e. $d_{\varphi}(a, b) \leq \max \left(d_{\varphi}(a, c), d_{\varphi}(c, b)\right)$ [25, p. 6]. If $\varphi: \mathrm{R} \rightarrow \mathbb{R}$ is an absolute value we denote by $\mathcal{T}_{\varphi}$ and $\mathcal{U}_{\varphi}$ the topology and the uniform structure induced by the corresponding metric $d_{\varphi}$. The open metric balls around $x \in \mathrm{R}$ with radius $0<\epsilon \in \mathbb{R}$ are denoted by $B_{\epsilon}^{\varphi}(x)$ and analogously we define $U_{\epsilon}^{\varphi}:=\left\{(x, y) \in \mathrm{R} \times \mathrm{R} \mid d_{\varphi}(x, y)<\epsilon\right\}$ for $0<\epsilon \in \mathbb{R}$. Then the metric balls $B_{\epsilon}^{\varphi}(x)$ and the $U_{\epsilon}^{\varphi}$ form a base for the topology $\mathcal{T}_{\varphi}$ and the uniform structure $\mathcal{U}_{\varphi}$.

In the case of an ordered field $\mathrm{R}$ we ask now for an absolute value $\varphi$ such that $\varphi: R \rightarrow \mathbb{R}$ is not only continuous with respect to $\mathcal{T}$ but such that $\mathcal{T}_{\varphi}=\mathcal{T}$ and $\mathcal{U}_{\varphi}=\mathcal{U}$. If this is the case we call the absolute value compatible with the ordering of $\mathrm{R}$.

Lemma 3.16 Let $\mathrm{R}$ be an ordered field and $\varphi: \mathrm{R} \rightarrow \mathbb{R}$ an absolute value. Then $\mathcal{T}=$ $\mathcal{T}_{\varphi} \Longleftrightarrow \mathcal{U}=\mathcal{U}_{\varphi}$.

ProOF: The inverse implication being trivial assume that $\mathcal{T}=\mathcal{T}_{\varphi}$. Then for all $0<\epsilon \in \mathbb{R}$ there exists $0<\delta \in \mathrm{R}$ such that $B_{\delta}(0) \subset B_{\epsilon}^{\varphi}(0)$. This implies $U_{\delta} \subset U_{\epsilon}^{\varphi}$ and vice versa.

If there is such a compatible absolute value then the topology $\mathcal{T}=\mathcal{T}_{\varphi}$ of $\mathrm{R}$ is first countable since it is induced by a metric and hence we can apply proposition 3.13. Furthermore $\varphi$ is continuous which implies that $\varphi$ is non-trivial. This implies that there are elements $\epsilon \in \mathrm{R}$ such that $0<\varphi(\epsilon)<1$ and hence $\varphi(\epsilon)^{n} \rightarrow 0$. Thus $\epsilon^{n} \rightarrow 0$ in the field $\mathrm{R}$ since the topologies $\mathcal{T}_{\varphi}$ and $\mathcal{T}$ are the same. Hence we have proved the following lemma:

Lemma 3.17 Let $\mathrm{R}$ be an ordered field and $\varphi: \mathrm{R} \rightarrow \mathbb{R}$ a compatible absolute value. Then there exists $0<\epsilon \in \mathrm{R}$ such that $\epsilon^{n} \rightarrow 0$.

At last we shall consider ordered fields and their real closure. A field $\mathrm{R}$ is called real closed iff it is ordered and any positive element has a square root in $\mathrm{R}$ and every polynomial of odd degree with coefficients in $\mathrm{R}$ has a root in $\mathrm{R}$ [21, p. 308]. Let $\mathrm{R}$ be an ordered field. An extension field $\widehat{R}$ of $R$ is called real closure of $R$ iff $\widehat{R}$ is real closed and algebraic over $R$ and the (unique) order of $\widehat{R}$ is an extension of the order in $R$ [22, p. 655]. Such a real closure exists for every orderd field and is unique up to isomorphisms [22, p. 656]. Furthermore for a real closed field one can prove the 'fundamental theorem of algebra': If $\mathrm{R}$ is a real closed field then the quadratic field extension $\mathrm{C}=\mathrm{R}(i)$ with $i^{2}:=-1$ is algebraically closed [21, p. 309].

For the quadratic field extension $\mathrm{C}=\mathrm{R}(i)$ of a real closed field $\mathrm{R}$ we define

$$
|z|:=\sqrt{z \bar{z}}=\sqrt{a^{2}+b^{2}} .
$$


for $z=a+i b \in \mathrm{C}$ where $a, b \in \mathrm{R}$ using the square root. Then $|z| \in \mathrm{R}$ and for the real elements $a \in \mathrm{R}$ this definition coincides with the previous definition since $\sqrt{a^{2}}=|a|$. Furthermore we have $|z|=0 \Longleftrightarrow z=0,|z w|=|z||w|$ and $|z+w| \leq|z|+|w|$ for $z, w \in C$ and hence $|\cdot|$ induces a topology on $\mathrm{C}$ which is compatible with the standard topology of the ordered field R. Again we will call this topology for $C$ the standard topology. Then the field $\mathrm{R}$ is Cauchy complete with respect to the standard topology iff its quadratic extension $\mathrm{C}$ is Cauchy complete with respect to the standard topology.

In the following we will give a generalization of the usual definition of a Hilbert space over $\mathbb{C}$ which leads to the definition of a Hilbert space over $\mathbf{C}=\mathrm{R}(i)$ where $\mathrm{R}$ is an ordered field which is Cauchy complete and real closed.

Let $\mathfrak{H}_{\mathrm{C}}$ be a pre-Hilbert space over $\mathrm{C}=\mathrm{R}(i)$ where $\mathrm{R}$ is a real closed field. In this case we can define a $\mathrm{R}$-norm for vectors in $\mathfrak{H}_{\mathrm{C}}$ that takes values in $\mathrm{R}$ by

$$
\|\phi\|:=\sqrt{\langle\phi, \phi\rangle}
$$

for $\phi \in \mathfrak{H}_{\mathbf{C}}$. Then we clearly have for $\phi, \psi \in \mathfrak{H}_{\mathbf{C}}$ and $a \in \mathrm{C}$ :

$$
\begin{gathered}
\|\phi\| \geq 0 \quad \text { and } \quad\|\phi\|=0 \Longleftrightarrow \phi=0 \\
\|a \phi\|=|a|\|\phi\| \\
\|\phi+\psi\| \leq\|\phi\|+\|\psi\| \\
\|\phi+\psi\|^{2}+\|\phi-\psi\|^{2}=2\|\phi\|^{2}+2\|\psi\|^{2}
\end{gathered}
$$

With this $\mathrm{R}$-norm the pre-Hilbert space $\mathfrak{H}_{\boldsymbol{C}}$ becomes a topological vector space where we define a base for the topology by the $\epsilon$-balls with respect to $\|\cdot\|$. Note that $\mathfrak{H}_{\mathrm{C}}$ is not a normed vector space in the usual sense since $\|\cdot\|$ takes values in $R$ and not in $\mathbb{R}$. An easy consequence is the following lemma:

Lemma 3.18 Let $\mathfrak{H}_{\mathrm{C}}$ be a pre-Hilbert space over $\mathrm{C}=\mathrm{R}(i)$ where $\mathrm{R}$ is a real closed field. Then the Hermitian product $\langle\cdot, \cdot\rangle: \mathfrak{H}_{\mathrm{C}} \times \mathfrak{H}_{\mathrm{C}} \rightarrow \mathrm{C}$ is continuous with respect to $\|\cdot\| \cdot$

By means of $\|\cdot\|$ we can define Cauchy sequences in $\mathfrak{H}_{\boldsymbol{c}}$ and this leads to the definition of a Hilbert space:

Definition 3.19 (Hilbert space) Let $\mathrm{C}=\mathrm{R}(i)$ be the quadratic field extension of a real closed field $\mathrm{R}$ and $\mathfrak{H}_{\mathrm{C}}$ a pre-Hilbert space over $\mathrm{C}$ with $\mathrm{R}$-norm $\|\cdot\|$. Then $\mathfrak{H}_{\mathrm{C}}$ is called a Hilbert space over $\mathrm{C}$ iff every Cauchy sequence in $\mathfrak{H}_{\mathrm{C}}$ converges in $\mathfrak{H}_{\mathrm{C}}$ with respect to $\|\cdot\|$.

Lemma 3.20 Let $\{0\} \neq \mathfrak{H}_{\mathrm{C}}$ be a Hilbert space over $\mathrm{C}=\mathrm{R}(i)$ where $\mathrm{R}$ is a real closed field. Then $\mathrm{C}$ and $\mathrm{R}$ are Cauchy complete.

Due to this lemma we should always assume that $\mathrm{C}$ is not only algebraically closed but also Cauchy complete if we want to consider Hilbert spaces over C. In a next step we want to construct the completion of a pre-Hilbert space to a Hilbert space.

Proposition 3.21 Let $\mathfrak{H}_{\mathrm{c}}$ be a pre-Hilbert space over $\mathrm{C}=\mathrm{R}(i)$ where $\mathrm{R}$ is real closed and Cauchy complete. Then up to unitary equivalence there is one Hilbert space $\widehat{\mathfrak{H C}_{\mathrm{C}}}$ such that there is an isometry $i: \mathfrak{H}_{\mathrm{C}} \rightarrow \widehat{\mathfrak{H}_{\mathrm{C}}}$ and $i\left(\mathfrak{H}_{\mathrm{C}}\right)$ is dense in $\widehat{\mathfrak{H}_{\mathrm{C}}}$. 
Let $\mathfrak{H}_{\mathrm{C}}$ and $\mathfrak{K}_{\mathrm{C}}$ be Hilbert spaces over $\mathrm{C}$ and $T: \mathfrak{H}_{\mathrm{C}} \rightarrow \mathfrak{K}_{\mathrm{C}}$ a linear map. Then clearly $T$ is continuous iff it is continuous at some point $\phi_{0} \in \mathfrak{H}_{\mathrm{C}}$ since $T$ is linear.

Lemma 3.22 Let $\mathfrak{H}_{\mathrm{C}}$ and $\mathfrak{K}_{\mathrm{C}}$ be Hilbert spaces over $\mathrm{C}=\mathrm{R}(i)$ where $\mathrm{R}$ is a real closed and Cauchy complete field. For a linear map $T: \mathfrak{H}_{\mathrm{C}} \rightarrow \mathfrak{K}_{\mathrm{C}}$ we have: $T$ is continuous iff there exists $C \in \mathrm{R}$ such that $\|T \phi\| \leq C\|\phi\|$ for all $\phi \in \mathfrak{H}_{\mathrm{C}}$ ( $T$ is bounded).

Remark: For continuous linear maps $T$ between Hilbert spaces over $\mathbb{C}$ one usually defines an operator norm by the supremum of $\|T \phi\| /\|\phi\|$ where $\phi \neq 0$ ranges over the Hilbert space. But in the case of a non-archimedian ordered field such a supremum does not exist in general though $T$ is bounded.

In the case that the standard topology of the field $\mathrm{R}$ is first countable we have the following important property of C-Hilbert spaces:

Lemma 3.23 Let $\mathfrak{H}_{\mathrm{C}}$ be a $\mathrm{C}$-Hilbert space where $\mathrm{C}=\mathrm{R}(i)$ and $\mathrm{R}$ is real closed, Cauchy complete and first countable. Let $W$ be a subspace of $\mathfrak{H}_{\mathrm{C}}$. If $W$ is dense in $\mathfrak{H}_{\mathrm{C}}$ then for any $\phi \in \mathfrak{H}_{\mathrm{C}}$ there exists a sequence $\phi_{n} \in W$ with $\phi_{n} \rightarrow \phi$.

An important concept in the usual theory of Hilbert spaces are the Hilbert bases. This leads to the following generalizations: Let $\mathfrak{H}_{\mathrm{C}}$ be a $\mathrm{C}$-Hilbert space where $\mathrm{C}=\mathrm{R}(i)$ and $\mathrm{R}$ is a real closed and Cauchy complete field. Let $\mathbb{I}$ be an index set and let $\mathcal{F}$ be the set of all finite subsets of $\mathbb{I}$. Let $\left\{e_{k}\right\}_{k \in \mathbb{I}}$ be a set of vectors in $\mathfrak{H}_{\boldsymbol{C}}$ then $\left\{e_{k}\right\}_{k \in \mathbb{I}}$ is called an orthonormal system iff $\left\langle e_{k}, e_{k^{\prime}}\right\rangle=\delta_{k k^{\prime}}$ for all $k, k^{\prime} \in \mathbb{I}$. In this case the set $\left\{e_{k}\right\}_{k \in \mathbb{I}}$ is clearly linear independent in $\mathfrak{H}_{\mathrm{C}}$. This leads to the definition of a Hilbert base:

Definition 3.24 (Hilbert base) Let $\mathfrak{H}_{\mathrm{C}}$ be a Hilbert space and let $\left\{e_{k}\right\}_{k \in \mathbb{I}}$ be an orthonormal set where $\mathrm{C}=\mathrm{R}(i)$ and $\mathrm{R}$ is real closed, Cauchy complete and first countable. Then $\left\{e_{k}\right\}_{k \in \mathbb{I}}$ is called a Hilbert base for $\mathfrak{H}_{\mathrm{C}}$ iff $\mathrm{C}-\operatorname{span}\left\{e_{k}\right\}_{k \in \mathbb{I}}$ is dense in $\mathfrak{H}_{\mathrm{C}}$ with respect to the topology induced by $\|\cdot\|$.

The following theorem is proved in a completely analogous fashion to the usual proofs in textbooks on functional analysis (see e.g. [34, p. 86])

Theorem 3.25 Let $\mathfrak{H}_{\mathrm{C}}$ be a $\mathrm{C}$-Hilbert space where $\mathrm{C}=\mathrm{R}(i)$ and $\mathrm{R}$ is a real closed and Cauchy complete field. Let $\left\{e_{k}\right\}_{k \in \mathbb{I}}$ be an orthonormal set. Then we have Bessel's inequality

$$
\sum_{k \in F}\left|\left\langle e_{k}, \phi\right\rangle\right|^{2} \leq\langle\phi, \phi\rangle \quad \forall \phi \in \mathfrak{H}_{\mathrm{C}}, \forall F \in \mathcal{F}
$$

and for all $\phi \in \mathfrak{H}_{\mathrm{C}}$ the following 'best approximation property'

$$
\left\|\phi-\sum_{k \in F} \alpha_{k} e_{k}\right\| \geq\left\|\phi-\sum_{k \in F}\left\langle e_{k}, \phi\right\rangle e_{k}\right\|
$$

for all $\alpha_{k} \in \mathrm{C}$ where the equality is only satisfied iff $\alpha_{k}=\left\langle e_{k}, \phi\right\rangle$. Now let in addition $\mathrm{R}$ be first countable, let $\mathbb{I}=\mathbb{N}$, and let $\left\{e_{k}\right\}_{k \in \mathbb{N}}$ be a Hilbert base for $\mathfrak{H}_{\mathrm{C}}$. Then there exists a sequence $\phi_{n}=\sum_{k=1}^{N_{n}} \alpha_{k}^{n} e_{k} \in \mathrm{C}$-span $\left\{e_{k}\right\}_{k \in \mathbb{I}}$ with $\phi_{n} \rightarrow \phi$ for any $\phi \in \mathfrak{H}_{\mathrm{C}}$ and

$$
\phi=\lim _{N \rightarrow \infty} \sum_{k=1}^{N}\left\langle e_{k}, \phi\right\rangle e_{k}=: \sum_{k=1}^{\infty}\left\langle e_{k}, \phi\right\rangle e_{k}
$$


and we have Parseval's equation:

$$
\|\phi\|^{2}=\sum_{k=1}^{\infty}\left|\left\langle e_{k}, \phi\right\rangle\right|^{2} \quad\langle\phi, \psi\rangle=\sum_{k=1}^{\infty}\left\langle\phi, e_{k}\right\rangle\left\langle e_{k}, \psi\right\rangle
$$

Moreover if $\alpha_{k} \in \mathrm{C}$ then $\sum_{k} \alpha_{k} e_{k}$ converges to a vector in $\mathfrak{H}_{\mathrm{C}}$ iff $\sum_{k}\left|\alpha_{k}\right|^{2}$ converges in $\mathrm{R}$. An orthonormal system $\left\{e_{k}\right\}_{k \in \mathbb{I}}$ is a Hilbert base for $\mathfrak{H}_{\mathrm{C}}$ iff Parseval's equation holds for any $\phi \in \mathfrak{H}_{\mathrm{C}}$.

As a (generic) example we will consider the $\ell^{2}$-space of a field $\mathrm{C}=\mathrm{R}(i)$ where $\mathrm{R}$ is real closed, Cauchy complete and first countable:

$$
\ell^{2}(\mathrm{C}):=\left\{\left(a_{k}\right)_{k \in \mathbb{N}} \mid a_{k} \in \mathrm{C} \text { such that } \sum_{k=1}^{\infty}\left|a_{k}\right|^{2} \text { converges in } \mathrm{R}\right\}
$$

together with the $\ell^{2}$-product

$$
\langle a, b\rangle:=\sum_{k=1}^{\infty} \bar{a}_{k} b_{k}
$$

Proposition 3.26 Let $\mathrm{C}=\mathrm{R}(i)$ be the quadratic field extension of $\mathrm{R}$ where $\mathrm{R}$ is real closed, Cauchy complete and first countable. Then $\ell^{2}(\mathrm{C})$ is a $\mathrm{C}$-Hilbert space and the vectors $\hat{e}_{k}:=$ $(0, \ldots, 0,1,0, \ldots)$ (where 1 is the $k$-th entry) form a countable Hilbert base for $\ell^{2}(\mathrm{C})$.

Proposition 3.27 Let $\mathfrak{H}_{\mathrm{C}}$ and $\mathfrak{K}_{\mathrm{C}}$ be Hilbert spaces where $\mathrm{C}=\mathrm{R}(i)$ and $\mathrm{R}$ is real closed, Cauchy complete and first countable. Let $\left\{e_{k}\right\}_{k \in \mathbb{I}}$ be a Hilbert base for $\mathfrak{H}_{\mathrm{C}}$.

i.) If $U: \mathfrak{H}_{\mathrm{C}} \rightarrow \mathfrak{K}_{\mathrm{C}}$ is an unitary map then $\left\{f_{k}\right\}_{k \in \mathbb{I}}$ with $f_{k}:=U e_{k}$ is a Hilbert base for $\mathfrak{K}_{\mathrm{C}}$.

ii.) If $\left\{f_{k}\right\}_{k \in \mathbb{I}}$ is a Hilbert base of $\mathfrak{K}_{\mathrm{C}}$ then there exists an unique unitary map $U: \mathfrak{H}_{\mathrm{C}} \rightarrow \mathfrak{K}_{\mathrm{C}}$ such that $U e_{k}=f_{k}$.

For later use we finally mention the following corollary:

Corollary 3.28 Let $\mathfrak{H}_{\mathrm{c}}$ be a pre-Hilbert space over $\mathrm{C}=\mathrm{R}(i)$ where $\mathrm{R}$ is real closed, Cauchy complete and first countable and let $\left\{e_{k}\right\}_{k \in \mathbb{I}}$ be an orthonormal system such that $\mathrm{C}$-span $\left\{e_{k}\right\}_{k \in \mathbb{I}}$ is dense in $\mathfrak{H}_{\mathrm{C}}$. Denote the completion of $\mathfrak{H}_{\mathrm{C}}$ by $\widehat{\mathfrak{H}_{\mathrm{C}}}$. Then $\left\{e_{k}\right\}_{k \in \mathbb{I}}$ is a Hilbert base of $\widehat{\mathfrak{H}_{\mathrm{C}}}$ and $\widehat{\mathfrak{H}_{\mathrm{C}}}$ is unitary equivalent to $\ell^{2}(\mathrm{C})$, i.e. there is an unitary map $U: \widehat{\mathfrak{H}_{\mathrm{C}}} \rightarrow \ell^{2}(\mathrm{C})$.

\section{The GNS Construction in deformation quantization}

Since the Laurent field $\mathbb{R}((\lambda)$ ) (definition (1)) is not real closed we are searching for a field extension of $\mathbb{R}((\lambda))$ such that this extension will be real closed and Cauchy complete with respect to the ordering relation since this is the important property we need if we want to consider Hilbert spaces as in the last section.

First we define the following systems of subsets of the rational numbers $\mathbb{Q}$ : 
Definition 4.1 $S \subset \mathbb{Q}$ is called NP-admissable iff $S$ has a smallest element and there is a positive integer such that $N \cdot S \subset \mathbb{Z}$ and $S$ is called CNP-admissable iff $S$ has a smallest element and $S \cap[i, j]$ is finite for any $i, j \in \mathbb{Q}$. Then we define $\mathcal{S}_{\mathrm{CNP}}:=\{S \in$ $\mathbb{Q} \mid S$ is CNP-admissable $\}$ and analogously $\mathcal{S}_{\mathrm{NP}}$.

Let $V$ be a vector space over $\mathbb{R}($ or $\mathbb{C}$ ) and $f: \mathbb{Q} \rightarrow V$. Then we define the support of $f$ by $\operatorname{supp} f:=\{q \in \mathbb{Q} \mid f(q) \neq 0\}$.

Definition 4.2 Let $V$ be a vector space over $\mathbb{R}$ (or $\mathbb{C}$ ). Then we define the formal NewtonPuiseux series (NP series) with coefficients in $V$ by

$$
V\left\langle\left\langle\lambda^{*}\right\rangle\right\rangle:=\left\{f: \mathbb{Q} \rightarrow V \mid \operatorname{supp} f \in \mathcal{S}_{\mathrm{NP}}\right\}
$$

and the completed Newton-Puiseux series (CNP series) with coefficients in $V$ by

$$
V\langle\langle\lambda\rangle\rangle:=\left\{f: \mathbb{Q} \rightarrow V \mid \operatorname{supp} f \in \mathcal{S}_{\mathrm{CNP}}\right\} .
$$

Clearly $V\left\langle\left\langle\lambda^{*}\right\rangle\right\rangle$ and $V\langle\langle\lambda\rangle\rangle$ are both vector spaces over $\mathbb{R}$ (or $\mathbb{C}$ ) and elements in $V\left\langle\left\langle\lambda^{*}\right\rangle\right\rangle$ and $V\langle\langle\lambda\rangle\rangle$ are written in the form

$$
f=\sum_{q \in \operatorname{supp} f} \lambda^{q} f_{q} \quad f_{q}:=f(q)
$$

and since $\mathcal{S}_{\mathrm{NP}} \subset \mathcal{S}_{\mathrm{CNP}}$ we notice that $V \subset V((\lambda)) \subset V\left\langle\left\langle\lambda^{*}\right\rangle\right\rangle \subset V\langle\langle\lambda\rangle\rangle$. If $f \in V\left\langle\left\langle\lambda^{*}\right\rangle\right\rangle$ then either the support is finite or it can be written as $f=\sum_{r=-M}^{\infty} \lambda^{\frac{r}{N}} f_{r}$ where $M \in \mathbb{Z}$ and $N \in \mathbb{N}$. If $f \in V\langle\langle\lambda\rangle\rangle$ then the support is again either finite or can be written as a sequence $q_{0}<q_{1}<\cdots \in \mathbb{Q}$ without any accumulation points. In both cases we will write

$$
f=\sum_{r=0}^{\infty} \lambda^{q_{r}} f_{q_{r}} .
$$

For $f \in V\langle\langle\lambda\rangle\rangle$ we define the order $o(f):=\min (\operatorname{supp} f)$ for $f \neq 0$ and set $o(0):=+\infty$ and we define

$$
\varphi(f):=2^{-o(f)} \text { for } f \neq 0 \quad \varphi(0):=0
$$

which leads to an ultra-metric $d_{\varphi}(f, g):=\varphi(f-g)$ for the vector space $V\langle\langle\lambda\rangle\rangle$.

Proposition 4.3 Let $V$ be a vector space over $\mathbb{R}$ (or $\mathbb{C})$. Then $V\langle\langle\lambda\rangle\rangle$ is a complete metric space with respect to the metric $d_{\varphi}$ and $V\left\langle\left\langle\lambda^{*}\right\rangle\right\rangle$ is dense in $V\langle\langle\lambda\rangle\rangle$.

Proof: Let $\left(f^{(n)}\right)_{n \in \mathbb{N}}$ be a Cauchy sequence in $V\langle\langle\lambda\rangle\rangle$ with respect to the metric $d_{\varphi}$. Then there are natural numbers $N_{0} \leq N_{1} \leq N_{2} \leq \cdots \in \mathbb{N}$ such that $d_{\varphi}\left(f^{(n)}, f^{(m)}\right)<2^{-k}$ for all $n, m \geq N_{k}$. Then we define $f_{q}:=f_{q}^{\left(N_{0}\right)}$ for $-\infty<q \leq 0$ and $f_{q}:=f_{q}^{\left(N_{k}\right)}$ for $k-1<q \leq k$ where $k \in \mathbb{N}$. Then $f=\sum_{q} \lambda^{q} f_{q}$ is a well-defined series in $V\langle\langle\lambda\rangle\rangle$ since supp $f$ has a smallest element and in any intervall of the form $[i, i+1]$ with $i \in \mathbb{Z}$ are only finitely many $f_{q} \neq 0$ for $q \in[i, i+1]$ and hence $\operatorname{supp} f \in \mathcal{S}_{\mathrm{CNP}}$. Since the $f^{(n)}$ coincide with $f$ up to a sufficiently increasing order thanks to the Cauchy condition it follows that $f^{(n)} \rightarrow f$ which implies that the metric space $V\langle\langle\lambda\rangle\rangle$ is complete. Now let $f=\sum_{q} \lambda^{q} f_{q}$ be an arbitrary element in $V\langle\langle\lambda\rangle\rangle$. Then every element of the sequence $f^{(m)}:=\sum_{q \leq m} \lambda^{q} f_{q}$ where $m \in \mathbb{N}$ has finite support and hence $f^{(m)} \in V\left\langle\left\langle\lambda^{*}\right\rangle\right\rangle$. Moreover clearly $f^{(m)} \rightarrow f$ which proves the proposition. 
Corollary 4.4 Let $V$ be a vector space over $\mathbb{R}$ (or $\mathbb{C}$ ) and $f=\sum_{q} \lambda^{q} f_{q} \in V\langle\langle\lambda\rangle$. Then $f^{(m)}:=\sum_{q \leq m} \lambda^{q} f_{q}$ converges to $f$.

We will now concentrate on the vector space $\mathbb{R}\left\langle\left\langle\lambda^{*}\right\rangle\right\rangle$ and $\mathbb{R}\langle\langle\lambda\rangle\rangle$ (and analogously for $\mathbb{C}\left\langle\left\langle\lambda^{*}\right\rangle\right\rangle$ and $\left.\mathbb{C}\langle\langle\lambda\rangle\rangle\right)$ itself. Analogously to the case of formal Laurent series we define a multiplication of two elements $a, b \in \mathbb{R}\langle\langle\lambda\rangle\rangle$ by

$$
a b=\left(\sum_{p \in \operatorname{supp} a} \lambda^{p} a_{p}\right)\left(\sum_{q \in \operatorname{supp} b} \lambda^{q} b_{q}\right):=\sum_{t \in \operatorname{supp} a+\operatorname{supp} b} \lambda^{t} \sum_{p+q=t} a_{p} b_{q}
$$

where $\operatorname{supp} a b=\operatorname{supp} a+\operatorname{supp} b:=\{p+q \mid p \in \operatorname{supp} a, q \in \operatorname{supp} b\}$. Then suppab $\in \mathcal{S}_{\mathrm{CNP}}$ and in any order $t \in \operatorname{supp} a b$ the sum $p+q=t$ for $p \in \operatorname{supp} a$ and $q \in \operatorname{supp} b$ is finite. Hence $a b$ is again a well-defined CNP series. A proof in a more general context could be found in [25, p. 81]. Moreover the product is clearly associative and commutative and we can find for any $0 \neq a \in \mathbb{R}\langle\langle\lambda\rangle\rangle$ an inverse $a^{-1} \in \mathbb{R}\langle\langle\lambda\rangle\rangle$ and hence $\mathbb{R}\langle\langle\lambda\rangle\rangle$ becomes a field. Furthermore one can show that $\mathbb{R}\left\langle\left\langle\lambda^{*}\right\rangle\right\rangle$ is a subfield of $\mathbb{R}\langle\langle\lambda\rangle\rangle$. A similar result holds for $\mathbb{C}\langle\langle\lambda\rangle\rangle$.

If $V$ is a vector space over $\mathbb{K}(\mathbb{K}=\mathbb{R}$ or $\mathbb{C})$ then $V\left\langle\left\langle\lambda^{*}\right\rangle\right\rangle$ is a vector space over $\mathbb{K}\left\langle\left\langle\lambda^{*}\right\rangle\right\rangle$ and $V\langle\langle\lambda\rangle\rangle$ is a vector space over $\mathbb{K}\langle\langle\lambda\rangle\rangle$. We call these vector spaces the canonical extensions of $V$ to vector spaces over the NP series and the CNP series with real (or complex) coefficients. Let $V^{*}$ be the (algebraic) dual vector space of $V$ then any $\mathbb{K}$-linear functional $\omega \in V^{*}$ has an obvious canonical $\mathbb{K}\langle\langle\lambda\rangle\rangle$-linear extension to a functional of $V\langle\langle\lambda\rangle\rangle$ by applying $\omega$ order by order. This extension will always be understood. A particular subspace of all $\mathbb{K}\langle\langle\lambda\rangle\rangle$-linear functionals $\omega: V\langle\langle\lambda\rangle\rangle \rightarrow \mathbb{R}\langle\langle\lambda\rangle\rangle$ is given by $V^{*}\langle\langle\lambda\rangle\rangle$, i.e. the linear functionals of the form $\omega=\sum_{q \in \operatorname{supp} \omega} \lambda^{q} \omega_{q}$ where $\omega_{q} \in V^{*}$ and $\operatorname{supp} \omega \in \mathcal{S}_{\mathrm{CNP}}$. Note that $V^{*}\langle\langle\lambda\rangle\rangle$ is in general a proper subspace of all $\mathbb{K}\langle\langle\lambda\rangle\rangle$-linear functionals of $V\langle\langle\lambda\rangle\rangle$, i.e. $V^{*}\langle\langle\lambda\rangle\rangle \subsetneq(V\langle\langle\lambda\rangle\rangle)^{*}$ iff $V$ is infinite-dimensional.

The field of CNP series $\mathbb{R}\langle\langle\lambda\rangle\rangle$ (or $\mathbb{C}\langle\langle\lambda\rangle\rangle$ ) has a canonical non-archimedian absolute value, namely $\varphi$ and $\mathbb{R}\langle\langle\lambda\rangle\rangle$ has an unique order which turns out to be compatible with the absolute value:

Proposition 4.5 The map $\varphi$ defined as in (39) is a non-archimedian and non-trivial absolute value for the fields $\mathbb{R}\langle\langle\lambda\rangle\rangle$ resp. $\mathbb{C}\langle\langle\lambda\rangle\rangle$. The field $\mathbb{R}\langle\langle\lambda\rangle\rangle$ is an ordered field and this unique order is compatible with the absolute value $\varphi$ and hence $\mathcal{T}=\mathcal{T}_{\varphi}$ and $\mathcal{U}=\mathcal{U}_{\varphi}$. The positive elements are given by $a>0$ iff $a_{q_{0}}>0$ where $q_{0}=\min (\operatorname{supp} a)$.

Theorem 4.6 i.) $\mathbb{C}\left\langle\left\langle\lambda^{*}\right\rangle\right\rangle \cong \mathbb{R}\left\langle\left\langle\lambda^{*}\right\rangle\right\rangle(i)\left(=\mathbb{R}\left\langle\left\langle\lambda^{*}\right\rangle\right\rangle \oplus i \mathbb{R}\left\langle\left\langle\lambda^{*}\right\rangle\right\rangle\right)$ is algebraically closed and $\mathbb{R}\left\langle\left\langle\lambda^{*}\right\rangle\right\rangle$ is real closed.

ii.) $\mathbb{C}\langle\langle\lambda\rangle\rangle \cong \mathbb{R}\langle\langle\lambda\rangle\rangle(i)(=\mathbb{R}\langle\langle\lambda\rangle\rangle \oplus i \mathbb{R}\langle\langle\lambda\rangle\rangle)$ is algebraically closed and $\mathbb{R}\langle\langle\lambda\rangle\rangle$ is real closed and both are Cauchy complete with respect to the ordering relation of $\mathbb{R}\langle\langle\lambda\rangle\rangle$.

Proof: The first part is the Newton-Puiseux theorem ([31, p. 61] or [22, p. 595] for a more general case). To prove the second part we notice that $\mathbb{C}\langle\langle\lambda\rangle\rangle$ is the completion of $\mathbb{C}\left\langle\left\langle\lambda^{*}\right\rangle\right\rangle$ with respect to the metric $d_{\varphi}$ induced by the absolute value $\varphi$ according to proposition 4.3 . Hence Kürschák's theorem ensures that $\mathbb{C}\langle\langle\lambda\rangle\rangle$ is again algebraically closed, see [22, p. 584]. 
Furthermore, since $\mathbb{C}\langle\langle\lambda\rangle\rangle \cong \mathbb{R}\langle\langle\lambda\rangle\rangle \oplus i \mathbb{R}\langle\langle\lambda\rangle\rangle$ it follows e.g. from the theorem of Artin and Schreier [22, p. 674] that $\mathbb{R}\langle\langle\lambda\rangle\rangle$ is real closed.

This theorem ensures that the fields $\mathbb{R}\langle\langle\lambda\rangle\rangle$ and $\mathbb{C}\langle\langle\lambda\rangle\rangle$ have indeed the algebraic and analytic properties needed for the GNS construction in deformation quantization and the definition of Hilbert spaces over $\mathbb{C}\langle\langle\lambda\rangle\rangle$. Note that the NP series would not suffice since they are not Cauchy complete. Using the field $\mathbb{C}\langle\langle\lambda\rangle\rangle$ we consider the $\mathbb{C}\langle\langle\lambda\rangle\rangle$-vector space $C^{\infty}(M)\langle\langle\lambda\rangle\rangle$ and extend the star product to a $\mathbb{C}\langle\langle\lambda\rangle\rangle$-bilinear product on $C^{\infty}(M)\langle\langle\lambda\rangle\rangle$. Hence the observable algebra in deformation quantization becomes a $\mathbb{C}\langle\langle\lambda\rangle\rangle$-algebra. To define states and GNS representations we need positive linear functionals of this algebra and first we notice that the results of section 2 not only hold for the field of formal Laurent series but also for the CNP case.

If we consider positive linear functionals of the particular form $\omega=\sum_{q \in \operatorname{supp} \omega}^{\infty} \lambda^{q} \omega_{q}$ where $\omega_{q}: C^{\infty}(M)\langle\langle\lambda\rangle\rangle \rightarrow \mathbb{C}$ and $\operatorname{supp} \omega \in \mathcal{S}_{\mathrm{CNP}}$ as mentioned above then we can show that for a state no negative powers in the formal parameter could occur using the Cauchy-Schwarz inequality for the state:

Lemma 4.7 Let $\omega: C^{\infty}(M)\langle\langle\lambda\rangle\rangle \rightarrow \mathbb{C}\langle\langle\lambda\rangle\rangle$ be a positive linear functional of the form $\omega=$ $\sum_{q \in \operatorname{supp} \omega}^{\infty} \lambda^{q} \omega_{q}$ such that $\omega(\mathbf{1})=1$. Then $q_{0}:=\min (\operatorname{supp} \omega)=0$ and $\omega_{0}$ is a classical state, i.e. $\omega_{0}(\bar{f} f) \geq 0$ for $f \in C^{\infty}(M)$.

ProOF: Let $f \in C^{\infty}(M)\langle\langle\lambda\rangle\rangle$ be a function such that $\omega_{q_{0}}(f) \neq 0$ where we can assume that $f \in C^{\infty}(M)$. Then $o(\omega(f) \omega(\bar{f}))=2 q_{0}$ and $\left.o(\bar{f} * f)\right) \geq q_{0}$ which is in contradiction to the Cauchy-Schwarz inequality $\omega(f) \omega(\bar{f}) \leq \omega(\bar{f} * f)$ if $q_{0}<0$ where we used $\omega(\mathbf{1})=1$. Moreover this implies $\omega_{q_{0}}(\bar{f} f) \geq 0$ since $\bar{f} * f=\bar{f} f+\ldots$

This lemma has the following physical interpretation: Not only the classical observable algebra $C^{\infty}(M)$ is deformed but also the classical states are deformed to obtain the states for the deformed algebra. Hence the restriction to these particular linear functionals in $\left(C^{\infty}(M)^{*}\right)\langle\langle\lambda\rangle\rangle$ fits well into the general concept of deformation. An interesting open problem seems to be the question whether $\left(C^{\infty}(M)^{*}\right)\langle\langle\lambda\rangle\rangle$ can be specified by some continuity properties.

This scheme opens up a lot of possibilities to study candidates for positive linear functionals from a purely geometrical point of view: one can always start with a classical measure $\omega_{0}$ having support in an interesting submanifold of the classical phase space. The simplest possibility consists in studying single points, that is the evaluation or Dirac delta functionals, which we shall do in the next two sections. One might also think about larger submanifolds such as certain energy surfaces of suitable classical Hamiltonian functions, or Lagrangean submanifolds. However, as we had already seen in section 2 the positivity of these functionals for the deformed algebra is in general no longer true, and higher order terms in the deformation parameter have to be added.

One might also think about a deformed version of statistical mechanics where $\omega_{0}$ is a classical statistical measure such as e.g. the Boltzmann measure $\exp \left(-\beta H_{0}\right)$ for a Hamiltonian function $H_{0}$ on the phase space such that the Liouville integral over this Boltzmann function converges. In a context where convergence in $\lambda$ in a distributional sense is (at least partially) assumed one can find some of these ideas already in [1, 2] where among other things KMS conditions are studied. 
At last we want to discuss the time development in the observable algebra and in a representation generated by some Hamiltonian. Let $H_{0} \in C^{\infty}(M)$ be a classical real Hamiltonian and let $h \in C^{\infty}(M)\langle\langle\lambda\rangle\rangle$ be a real CNP series with $o(h)>0$ and define $H:=H_{0}+h$. Then the Heisenberg equation of motion with respect to the Hamiltonian $H$ is given by

$$
\frac{d}{d t} f_{t}=\frac{1}{i \lambda}\left[f_{t}, H\right]=\frac{1}{i \lambda}\left(f_{t} * H-H * f_{t}\right)
$$

where $t \mapsto f_{t} \in C^{\infty}(M)\langle\langle\lambda\rangle\rangle$ is the trajectory through $f_{0} \in C^{\infty}(M)\langle\langle\lambda\rangle\rangle$ and $t \in \mathbb{R}$. We ask now for solutions of (32) for a given initial condition $f_{0}$. Let $X_{H_{0}}$ be the Hamiltonian vector field of $H_{0}$ and assume for simplicity that $X_{H_{0}}$ is complete. In this case the flow $\phi_{t}: M \rightarrow M$ of $X_{H_{0}}$ is a one-parameter group of symplectic diffeomorphisms of M.

Proposition 4.8 With the above notations we have: The Heisenberg equation of motion has an unique solution $f_{t}$ defined for all $t \in \mathbb{R}$ for any given initial condition $f_{0} \in C^{\infty}(M)\langle\langle\lambda\rangle\rangle$ and $f_{t}$ is given by

$$
f_{t}=\phi_{t}^{*} \circ \mathcal{T} \exp \left(\int_{0}^{t} \phi_{-\tau}^{*} \circ \widehat{H} \circ \phi_{\tau}^{*} d \tau\right) f_{0}
$$

where $\mathcal{T}$ exp means the time ordered exponential and $\widehat{H}(g):=\frac{1}{i \lambda}[g, H]-\left\{g, H_{0}\right\}$ satisfies $o(\widehat{H}(g))>o(g)$ for all $g \in C^{\infty}(M)\langle\langle\lambda\rangle\rangle$.

Remarks: Fedosov has proved a similar result in [16, Sec. 5.4.]. In the case where $X_{H_{0}}$ is not complete one can prove the existence and uniqueness of solutions of (32) for example for initial conditions $f_{0}$ with compact support and sufficiently small times. We will denote the solutions of (32) in the symbolical way

$$
f_{t}=e^{\frac{i t}{\lambda} \operatorname{ad}(H)} f_{0}
$$

where $\operatorname{ad}(H) f:=[H, f]$.

The time development of the states is given by a Schrödinger equation but to formulate this equation we have to specify a representation of the observable algebra. We consider a positive linear functional $\omega$ of $C^{\infty}(M)\langle\langle\lambda\rangle\rangle$ and the related GNS representation $\pi_{\omega}$ on $\mathfrak{H}_{\omega}:=C^{\infty}(M)\langle\langle\lambda\rangle\rangle / \mathcal{J}_{\omega}$. Then the Schrödinger equation is given by

$$
i \lambda \frac{d}{d t} \psi(t)=\pi_{\omega}(H) \psi(t)
$$

where $\psi(t) \in \mathfrak{H}_{\omega}$. Specifying an initial condition $\psi(0)$ one can rewrite the Schrödinger equation into an integral equation

$$
\psi(t)=\psi(0)+\frac{1}{i \lambda} \int_{0}^{t} \pi_{\omega}(H) \psi(\tau) d \tau
$$

but now iterating this equation will generate in general arbitrarily high negative powers of $\lambda$ and hence it will not converge in the formal sense! A rather simple example is provided by any real multiple of the constant function 1 .

But under certain conditions the well-defined time development of the observables can be used to find solutions of (34): 
Theorem 4.9 Let $\omega: C^{\infty}(M)\langle\langle\lambda\rangle\rangle \rightarrow \mathbb{C}\langle\langle\lambda\rangle\rangle$ be a positive linear functional and $H=$ $H_{0}+h \in C^{\infty}(M)\langle\langle\lambda\rangle\rangle$ as above. If $H$ is contained in the Gel'fand ideal $\mathcal{J}_{\omega}$ of $\omega$ then the Schrödinger equation (34) has an unique solution for $t \in \mathbb{R}$ and any initial condition $\psi(0) \in \mathfrak{H}_{\omega}$. Moreover if $f \in C^{\infty}(M)\langle\langle\lambda\rangle\rangle$ and $f_{t}$ is the solution of (39) with initial condition $f$ then

$$
\psi(t):=\psi_{f_{-t}}=\psi_{\exp \left(-\frac{i t}{\lambda} \operatorname{ad}(H)\right) f}
$$

is the unique solution with initial condition $\psi(0)=\psi_{f}$.

ProOF: Let $f_{t}$ be the solution of (32) with initial condition $f$. Since $H \in \mathcal{J}_{\omega}$ we have

$$
\frac{d}{d t} \psi(t)=\frac{d}{d t} f_{-t} \bmod \mathcal{J}_{\omega}=-\frac{1}{i \lambda}\left(f_{-t} * H-H * f_{-t}\right) \bmod \mathcal{J}_{\omega}=\frac{1}{i \lambda} \pi_{\omega}(H) \psi(t) .
$$

Remarks: With the GNS representation we can define a time development of the states in a purely formal way without considering any convergence properties for $\lambda=\hbar$. There are other methods to define a time development as for example the star exponential [4] which is the starting point for a spectral analysis. In this approach the time development operator is viewed as a certain distribution if one substitutes the formal parameter $\lambda$ by $\hbar \in \mathbb{R}$.

If $\omega$ is a state then the condition $H \in \mathcal{J}_{\omega}$ implies that the vacuum vector $\psi_{\mathbf{1}}$ is invariant under the time development and hence $\omega$ could be called an invariant state under $H$. The search for invariant states under certain group actions is a well-studied problem in algebraic quantum field theory [20]. In our approach it raises the question whether there is a positive linear functional such that the left ideal generated by some Hamiltonian is contained in the Gel'fand ideal of that functional. This condition would determine a preferred choice of a state if one wants to consider the quantum theory of a certain classical Hamiltonian.

\section{Example I: The Wick product and $\delta$-functionals in $\mathbb{C}^{n}$}

We consider the Wick product (see for example [7]) in $\mathbb{C}^{n}$ which is defined for $f, g \in$ $C^{\infty}\left(\mathbb{C}^{n}\right)\langle\langle\lambda\rangle\rangle$ by

$$
f * g:=\sum_{r=0}^{\infty} \frac{(2 \lambda)^{r}}{r !} \frac{\partial^{r} f}{\partial z^{i_{1}} \cdots \partial z^{i_{r}}} \frac{\partial^{r} g}{\partial \bar{z}^{i_{1}} \cdots \partial \bar{z}^{i_{r}}}
$$

where $z^{1}, \ldots, z^{n}$ are the canonical holomorphic coordinates in $\mathbb{C}^{n}$ and summation over repeated indices is always understood. Moreover we consider the evaluation functional ('deltafunctional') $\delta_{p}$ at the point $p \in \mathbb{C}^{n}$

$$
\delta_{p}[f]:=f(p)
$$

as a $\mathbb{C}\langle\langle\lambda\rangle\rangle$-linear functional $\delta_{p}: C^{\infty}\left(\mathbb{C}^{n}\right)\langle\langle\lambda\rangle\rangle \rightarrow \mathbb{C}\langle\langle\lambda\rangle\rangle$. Then $\delta_{p}$ turns out to be positive with respect to the Wick product:

Lemma 5.1 Let $p \in \mathbb{C}^{n}$. Then $\delta_{p}$ is a positive linear functional with respect to the Wick product (35). Moreover it is clearly a state. The Gel'fand ideal of $\delta_{p}$ is given by

$$
\mathcal{J}_{p}:=\left\{f \in C^{\infty}\left(\mathbb{C}^{n}\right)\langle\langle\lambda\rangle\rangle \mid \frac{\partial^{|I|} f}{\partial \bar{z}^{I}}(p)=0 \text { for all multiindices } I\right\} .
$$


This lemma is proved by observing that $\bar{f} * f$ evaluated at a point is just a series of nonnegative elements in $\mathbb{R}\langle\langle\lambda\rangle\rangle$. Using Borel's lemma [33] we easily find the following isomorphism:

Proposition 5.2 We have the following isomorphism of $\mathbb{C}\langle\langle\lambda\rangle\rangle$-vector spaces

$$
\mathfrak{H}_{p}:=C^{\infty}\left(\mathbb{C}^{n}\right)\langle\langle\lambda\rangle\rangle / \mathcal{J}_{p} \cong\left(\mathbb{C}\left[\left[\bar{y}^{1}, \ldots, \bar{y}^{n}\right]\right]\right)\langle\langle\lambda\rangle\rangle
$$

and an isomorhism is given by the formal $\bar{z}$-Taylor series at $p$

$$
\mathfrak{H}_{p} \ni \psi_{f} \mapsto \sum_{r=0}^{\infty} \frac{1}{r !} \frac{\partial^{r} f}{\partial \bar{z}^{i_{1}} \cdots \partial \bar{z}^{i_{r}}}(p) \bar{y}^{i_{1}} \cdots \bar{y}^{i_{r}}
$$

and the Hermitian product induced by $\delta_{p}$ is given by

$$
\left\langle\psi_{f}, \psi_{g}\right\rangle=\sum_{r=0}^{\infty} \frac{(2 \lambda)^{r}}{r !} \overline{\frac{\partial^{r} f}{\partial \bar{z}^{i_{1}} \cdots \partial \bar{z}^{i_{r}}}(p)} \frac{\partial^{r} g}{\partial \bar{z}^{i_{1}} \cdots \bar{z}^{i_{r}}}(p) .
$$

In the following we shall identify $\psi_{f}$ with the formal $\bar{z}$-Taylor series and consider only $p=0$ for simplicity. Then we can determine the GNS representation $\pi_{0}$ induced by $\delta_{0}$ by calculating the formal $\bar{z}$-Taylor series of $f * g$ in order to obtain $\pi_{0}(f) \psi_{g}$.

Lemma 5.3 (The formal Wick representation) For $f \in C^{\infty}\left(\mathbb{C}^{n}\right)\langle\langle\lambda\rangle\rangle$ we find

$$
\pi_{0}(f)=\sum_{r, s=0}^{\infty} \frac{(2 \lambda)^{r}}{r ! s !} \frac{\partial^{r+s} f}{\partial z^{i_{1}} \cdots \partial z^{i_{r}} \partial \bar{z}^{j_{1}} \cdots \partial \bar{z}^{j_{s}}}(0) \bar{y}^{j_{1}} \cdots \bar{y}^{j_{s}} \frac{\partial}{\partial \bar{y}^{i_{1}}} \cdots \frac{\partial}{\partial \bar{y}^{i_{r}}}
$$

In particular we have for polynomials the Wick ordering (normal ordering):

$$
\pi_{0}\left(z^{i_{1}} \cdots z^{i_{r}} \bar{z}^{j_{1}} \cdots \bar{z}^{j_{s}}\right)=(2 \lambda)^{r} \bar{y}^{j_{1}} \cdots \bar{y}^{j_{s}} \frac{\partial}{\partial \bar{y}^{i_{1}}} \cdots \frac{\partial}{\partial \bar{y}^{i_{r}}}
$$

Now we will consider the completion of $\mathfrak{H}_{0}$ to a $\mathbb{C}\langle\langle\lambda\rangle\rangle$-Hilbert space.

Proposition 5.4 The completion $\widehat{\mathfrak{H}_{0}}$ of $\mathfrak{H}_{0}$ is given by

$$
\begin{aligned}
\widehat{\mathfrak{H}_{0}}= & \left\{\phi=\sum_{r=0}^{\infty} \frac{1}{r !} a_{i_{1} \cdots i_{r}}(\lambda) \bar{y}^{i_{1}} \cdots \bar{y}^{i_{r}} \mid a_{i_{1} \cdots i_{r}}(\lambda) \in \mathbb{C}\langle\langle\lambda\rangle\rangle\right. \\
& \text { such that } \left.\sum_{r=0}^{\infty} \frac{(2 \lambda)^{r}}{r !} \overline{a_{i_{1} \cdots i_{r}}(\lambda)} a_{i_{1} \cdots i_{r}}(\lambda) \text { converges in } \mathbb{C}\langle\langle\lambda\rangle\rangle\right\} .
\end{aligned}
$$

Let $K=\left(k_{1}, \ldots, k_{n}\right)$ be a multiindex then the vectors

$$
e_{K}:=\frac{1}{\sqrt{\lambda^{|K|} K !}}\left(\bar{y}^{1}\right)^{k_{1}} \cdots\left(\bar{y}^{n}\right)^{k_{n}} \in \widehat{\mathfrak{H}_{0}} \quad \text { where } \begin{gathered}
|K|:=k_{1}+\cdots+k_{n} \\
K !:=k_{1} ! \cdots k_{n} !
\end{gathered}
$$

form a Hilbert base for $\widehat{\mathfrak{H}_{0}}$ and hence $\widehat{\mathfrak{H}_{0}}$ is isometric to $\ell^{2}(\mathbb{C}\langle\langle\lambda\rangle\rangle)$. 
Remark: An element in $\mathfrak{H}_{0}$ has a lowest order in $\lambda$ according to equation (38) but the coefficients $a_{i_{1} \cdots i_{r}}(\lambda)$ of an element in $\widehat{\mathfrak{H}_{0}}$ written as in (43) could have decreasing orders in $\lambda$. Assigning to $\bar{y}^{1}, \ldots, \bar{y}^{n}$ the total degree 1 and to $\lambda$ the total degree 2 the elements in $\widehat{\mathfrak{H}_{0}}$ could be understood as formal CNP series in this total degree.

As an example we want to consider the harmonic oszillator with classical Hamiltonian $H:=\frac{1}{2} \omega|z|^{2}$ where $\omega \in \mathbb{R}^{+}$is the oszillator frequency. First we notice that $H$ is an element of the Gel'fand ideal $\mathcal{J}_{0}$ and $\pi_{0}(H)=\lambda \omega \bar{y}^{k} \partial / \partial \bar{y}^{k}$. Note that this operator is clearly defined on all of $\widehat{\mathfrak{H}_{0}}$ as a bounded and hence continuous operator. We can ask for its spectrum in the following sense:

$$
\operatorname{spec}\left(\pi_{0}(H)\right):=\left\{\mu \in \mathbb{C}\langle\langle\lambda\rangle\rangle \mid\left(\pi_{0}(H)-\mu \mathbf{1}\right) \text { is bijective }\right\}
$$

By an easy calculation we find that the spectrum is purely discrete, namely the well-known oszillator spectrum:

$$
\operatorname{spec}\left(\pi_{0}(H)\right)=\{0, \lambda \omega, 2 \lambda \omega, \ldots\}
$$

Moreover we notice that the vectors $e_{K}$ are eigenvectors to the eigenvalue $\lambda \omega|K|$ and hence they are a Hilbert base of eigenvectors to the harmonic oszillator.

At last we want to discuss a way how one can get back convergence in $\lambda$ if we substitute $\lambda$ by $\hbar \in \mathbb{R}$. The main idea is that we ask for convergence in the representation and not in the algebra. We define for a fixed $\hbar \in \mathbb{R}^{+}$

$$
\begin{aligned}
& H(\hbar):=\left\{\phi \in \widehat{\mathfrak{H}_{0}}\left|\sum_{r=0}^{\infty} \hbar^{q_{r}} a_{q_{r}}^{(K)}:=\left\langle e_{K}, \phi\right\rangle\right|_{\lambda=\hbar} \text { converges absolutely } \forall K\right. \\
&\text { and } \left.\sum_{|K|=0}^{\infty}\left(\sum_{r=0}^{\infty} \hbar^{q_{r}}\left|a_{q_{r}}^{(K)}\right|\right)^{2}<\infty\right\} \\
& N(\hbar):=\left\{\phi \in \widehat{\mathfrak{H}_{0}}\left|\left\langle e_{K}, \phi\right\rangle\right|_{\lambda=\hbar}=0\right\} .
\end{aligned}
$$

Lemma 5.5 Let $\phi=\sum_{r=0}^{\infty} \frac{1}{r !} a_{i_{1} \ldots i_{r}}(\lambda) \bar{y}^{i_{1}} \cdots \bar{y}^{i_{r}} \in \widehat{\mathfrak{H}_{0}}$. If $\phi \in H(\hbar)$ then we have:

i.) $a_{i_{1} \ldots i_{r}}(\lambda)$ converges absolutely for $\lambda=\hbar$.

ii.) The Hermitian product $\langle\phi, \phi\rangle$ is absolutely convergent for $\lambda=\hbar$ and positive semidefinite in $\mathbb{C}$ :

$$
\left.\langle\phi, \phi\rangle\right|_{\lambda=\hbar}=\sum_{r=0}^{\infty} \frac{(2 \hbar)^{r}}{r !} \overline{a_{i_{1} \ldots i_{r}}(\hbar)} a_{i_{1} \ldots i_{r}}(\hbar) \geq 0
$$

iii.) $H(\hbar)$ is a $\mathbb{C}$-vector space and $N(\hbar)$ is a $\mathbb{C}$-subvector space of $H(\hbar)$. For $\phi, \psi \in H(\hbar)$ the Hermitian product $\langle\phi, \psi\rangle$ converges absolutely in $\mathbb{C}$ for $\lambda=\hbar$.

iv.) $F(z):=\sum_{r=0}^{\infty} \frac{a_{i_{1} \ldots i_{r}}(\hbar)}{r !} \bar{z}^{i_{1}} \ldots \bar{z}^{i_{r}}$ is an entire anti-holomorphic function on $\mathbb{C}^{n}$. 
Part ii.) and iii.) will be proved in a more general context in theorem 6.3. Let $\phi$ be given as in the above lemma and let $\psi=\sum_{r=0}^{\infty} \frac{1}{r !} b_{i_{1} \ldots i_{r}}(\lambda) \bar{y}^{i_{1}} \cdots \bar{y}^{i_{r}}$ be another element in $H(\hbar)$. Then we have the following theorem:

Theorem 5.6 The quotient $\mathbb{C}$-vector space $\mathcal{H}(\hbar):=H(\hbar) / N(\hbar)$ with the Hermitian product

$$
\langle\phi \bmod N(\hbar), \psi \bmod N(\hbar)\rangle:=\sum_{r=0}^{\infty} \frac{(2 \hbar)^{r}}{r !} \overline{a_{i_{1} \ldots i_{r}}(\hbar)} b_{i_{1} \ldots i_{r}}(\hbar)
$$

is a Hilbert space over $\mathbb{C}$ which is isometric to the Hilbert space of anti-holomorphic functions $f: \mathbb{C}^{n} \rightarrow \mathbb{C}$

$$
\mathcal{H}(\hbar) \cong\left\{f:\left.\mathbb{C}^{n} \rightarrow \mathbb{C}\left|\sum_{r=0}^{\infty} \frac{(2 \hbar)^{r}}{r !} \sum_{i_{1}, \ldots, i_{r}}\right| \frac{\partial^{r} f}{\partial \bar{z}^{i_{1}} \cdots \partial \bar{z}^{i_{r}}}(0)\right|^{2}<\infty\right\}
$$

with the Gaussian product

$$
\langle f, g\rangle=\frac{1}{(2 \pi \hbar)^{n}} \int_{\mathbb{C}^{n}} e^{-\frac{|z|^{2}}{2 \hbar}} \overline{f(z)} g(z) d z^{1} \cdots d z^{n} d \bar{z}^{1} \cdots d \bar{z}^{n},
$$

and the canonical isometry is given by $\phi \mapsto F$ where $F$ is the anti-holomorphic function defined by $\phi$ as in lemma $5.5 \mathrm{iv}$.$) .$

Moreover the GNS representation of $C^{\infty}\left(\mathbb{C}^{n}\right)\langle\langle\lambda\rangle\rangle$ induces a representation $\mathcal{Q}$ on the $\mathbb{C}$ Hilbert space $\mathcal{H}(\hbar)$ at least for 'many' functions. We consider only those elements $f \in$ $C^{\infty}\left(\mathbb{C}^{n}\right)\langle\langle\lambda\rangle\rangle$ with $\pi_{0}(f) N(\hbar) \subseteq N(\hbar)$. Then we define

$$
D_{f}(\hbar):=\left\{\phi \in H(\hbar) \mid \pi_{0}(f) \phi \in H(\hbar)\right\} ; \quad \mathcal{D}_{f}(\hbar):=D_{f}(\hbar) / N(\hbar)
$$

and call $\mathcal{D}_{f}(\hbar) \subseteq \mathcal{H}(\hbar)$ the domain of $f$ since we can define an operator $\mathcal{Q}(f)$ with domain of definition $\mathcal{D}_{f}(\hbar)$ by

$$
\mathcal{Q}(f)(\phi \bmod N(\hbar)):=\pi_{0}(f) \phi \bmod N(\hbar) \quad \text { for } \phi \in D_{f}(\hbar) .
$$

In general $\mathcal{D}_{f}(\hbar)$ may be very small but there are 'many' functions (i.e. all polynomials in $z$ and $\bar{z})$ such that $\mathcal{D}_{f}(\hbar)$ is dense in $\mathcal{H}(\hbar)$ or even equal to $\mathcal{H}(\hbar)$. In this case we clearly have

$$
\mathcal{Q}(f * g)=\mathcal{Q}(f) \circ \mathcal{Q}(g)
$$

and hence we call $\mathcal{Q}$ a quantization map. If we write $\mathcal{H}(\hbar)$ in the form (49) this is of course just the well-known Bargmann quantization.

\section{General results for Kähler manifolds}

In this section we shall derive some general results for Kähler manifolds with the Fedosov star product of Wick type which had been constructed in [9]. First we define the formal Wick algebra in $2 n$ parameters by

$$
\mathcal{W}_{n}:=\left(\mathbb{C}\left[\left[y^{1}, \ldots, y^{n}, \bar{y}^{1}, \ldots, \bar{y}^{n}\right]\right]\right)\langle\langle\lambda\rangle\rangle
$$


then $\mathcal{W}_{n}$ is clearly a $\mathbb{C}\langle\langle\lambda\rangle\rangle$-vector space and we define a pointwise multiplication of the formal power series in $y^{1}, \ldots, \bar{y}^{n}$ as usual. Then $\mathcal{W}_{n}$ becomes an associative and commutative algebra over $\mathbb{C}\langle\langle\lambda\rangle\rangle$ with unit element. Moreover $\mathcal{W}_{n}$ becomes a ${ }^{*}$-algebra if we extend the complex conjugation such that $y^{k}$ is mapped to $\bar{y}^{k}$ and vice versa. This formal Wick algebra could be deformed by an analogue of the usual Wick product. We define

$$
a \circ b:=\sum_{r=0}^{\infty} \frac{(2 \lambda)^{r}}{r !} \frac{\partial^{r} a}{\partial y^{i_{1}} \cdots \partial y^{i_{r}}} \frac{\partial^{r} b}{\partial \bar{y}^{i_{1}} \cdots \partial \bar{y}^{i_{r}}}
$$

for $a, b \in \mathcal{W}_{n}$. This leads to an associative deformation of the pointwise multiplication such that the complex conjugation is still an antilinear algebra involution and $a \circ 1=1 \circ a=a$. Every element $a \in \mathcal{W}_{n}$ could be written as

$$
a=\sum_{r, s=0}^{\infty} \frac{1}{r ! s !} a_{i_{1} \ldots i_{r} j_{1} \ldots j_{s}} y^{i_{1}} \cdots y^{i_{r}} \bar{y}^{j_{1}} \cdots \bar{y}^{j_{s}} .
$$

Then we define the delta functional as the projection onto the part without explicit powers of the formal parameters $y^{1}, \ldots, \bar{y}^{n}$ :

$$
\delta(a):=a_{00} \in \mathbb{C}\langle\langle\lambda\rangle\rangle
$$

This will also be written in the following symbolical way: $\delta(a)=a_{00}=\left.a\right|_{y=0}$. Then it is easy to see that the delta functional is a positive linear functional:

Proposition 6.1 i.) The delta functional $\delta: \mathcal{W}_{n} \rightarrow \mathbb{C}\langle\langle\lambda\rangle\rangle$ is a state of $\mathcal{W}_{n}$ with respect to the Wick product and the Gel'fand ideal of $\delta$ is given by:

$$
\mathcal{J}=\left\{a \in \mathcal{W}_{n}\left|\frac{\partial^{|I|} a}{\partial \bar{y}^{I}}\right|_{y=0}=0 \text { for all multiindices } I\right\}
$$

ii.) The quotient space $\mathcal{W}_{n} / \mathcal{J}$ is isomorphic to $\mathfrak{H}_{n}^{\prime}:=\left(\mathbb{C}\left[\left[\bar{y}^{1}, \ldots, \bar{y}^{n}\right]\right]\right)\langle\langle\lambda\rangle\rangle$ with the 'formal $\bar{y}$-Taylor series' as isomorphism

$$
\left.a \bmod \mathcal{J} \mapsto \sum_{s=0}^{\infty} \frac{1}{s !} \frac{\partial^{s} a}{\partial \bar{y}^{i_{1}} \cdots \partial \bar{y}^{i_{s}}}\right|_{y=0} \bar{y}^{i_{r}} \cdots \bar{y}^{i_{s}}
$$

and the Hermitian product induced by $\delta$ is given by

$$
\left\langle\sum_{r=0}^{\infty} \frac{1}{r !} a_{i_{1} \ldots i_{r}} \bar{y}^{i_{r}} \ldots \bar{y}^{i_{r}}, \sum_{s=0}^{\infty} \frac{1}{s !} b_{j_{1} \ldots j_{s}} \bar{y}^{j_{r}} \ldots \bar{y}^{j_{s}}\right\rangle=\sum_{r=0}^{\infty} \frac{(2 \lambda)^{r}}{r !} \overline{a_{i_{1} \ldots i_{r}}} b_{i_{1} \ldots i_{r}}
$$

The properties of $\mathfrak{H}_{n}^{\prime}$ and its completion $\mathfrak{H}_{n}$ were already discussed in proposition 5.4 where $\mathfrak{H}_{n}^{\prime} \cong \mathfrak{H}_{0}$.

Now we want to investigate the convergence of the Hermitian products in a Hilbert space $\mathfrak{H}$ over $\mathbb{C}\langle\langle\lambda\rangle\rangle$ with a countable Hilbert base $\left\{e_{k}\right\}_{k \in \mathbb{N}}$. As we will see in section 7 a $\mathbb{C}\langle\langle\lambda\rangle\rangle$ Hilbert space has in general no Hilbert base but we will see important examples if we consider Kähler manifolds. 
First we will mention some easy convergence properties of CNP series. Let $\mathbb{C}^{-}:=\mathbb{C} \backslash\{x \in$ $\mathbb{R} \mid x \leq 0\}$ and $B_{r}^{-}:=B_{r}(0) \cap \mathbb{C}^{-}$where $B_{r}(0)$ is the open disk around $0 \in \mathbb{C}$ of radius $r$. For $q \in \mathbb{Q}$ let $z^{q}$ be the holomorphic root defined on $\mathbb{C}^{-}$. For a formal CNP series $a=$ $\sum_{r=0}^{\infty} \lambda^{q_{r}} a_{q_{r}} \in \mathbb{C}\langle\langle\lambda\rangle\rangle$ we define the radius of convergence by $R:=\sup _{t \in \mathbb{R}^{+}}\left\{\sum_{r=0}^{\infty} t^{q_{r}}\left|a_{q_{r}}\right|<\right.$ $\infty\}$ then we can prove the following proposition:

Proposition 6.2 Let $a \in \mathbb{C}\langle\langle\lambda\rangle\rangle$ with convergence radius $R>0$. Then $f(z):=\sum_{r=0}^{\infty} z^{q_{r}} a_{q_{r}}$ converges absolutely and normally in $B_{R}^{-}$. Hence $f: z \in B_{R}^{-} \rightarrow f(z) \in \mathbb{C}$ is a holomorphic function.

Now we define $H(\hbar)$ and $N(\hbar)$ in the following way:

$$
\begin{aligned}
H(\hbar):= & \left\{\phi \in \mathfrak{H}\left|\forall k \in \mathbb{N}:\left\langle e_{k}, \phi\right\rangle\right|_{\lambda=\hbar}=\sum_{r=0}^{\infty} \hbar^{q_{r}} a_{q_{r}}^{(k)}\right. \text { converges absolutely } \\
& \text { and } \left.\sum_{r=0}^{\infty}\left|F_{k}(\hbar)\right|^{2}<\infty \text { where } F_{k}(\hbar):=\sum_{r=0}^{\infty} \hbar^{q_{r}}\left|a_{q_{r}}^{(k)}\right|\right\} \\
N(\hbar):= & \left\{\phi \in H(\hbar)\left|\forall k \in \mathbb{N}:\left\langle e_{k}, \phi\right\rangle\right|_{\lambda=\hbar}=0\right\}
\end{aligned}
$$

First we notice that $F_{k}(\hbar)$ is well-defined and extends to a holomorphic function $F_{k}$ on $B_{\hbar}^{-}$. Using Cauchy's theorem for iterated series and Weierstraß' theorem for convergence of holomorphic functions we easily get the following theorem:

Theorem 6.3 Let $\mathfrak{H}$ be a $\mathbb{C}\langle\langle\lambda\rangle\rangle$-Hilbert space with countable Hilbert base $\left\{e_{k}\right\}_{k \in \mathbb{N}}$ and let $H(\hbar)$ and $N(\hbar)$ be defined as above and $\phi, \psi \in H(\hbar)$.

i.) $H(\hbar)$ is a $\mathbb{C}$-vector space and $N(\hbar)$ is a $\mathbb{C}$-subvector space of $H(\hbar)$.

ii.) The Hermitian product of $\mathfrak{H}$ induces a semidefinite sesquilinear form on $H(\hbar)$ by

$$
\left.\langle\phi, \psi\rangle\right|_{\lambda=\hbar}=\langle\phi, \psi\rangle_{\hbar}:=\left.\left.\sum_{k=1}^{\infty}\left\langle\phi, e_{k}\right\rangle\right|_{\lambda=\hbar}\left\langle e_{k}, \psi\right\rangle\right|_{\lambda=\hbar}
$$

and $\langle\phi, \phi\rangle_{\hbar}=0$ iff $\phi \in N(\hbar)$.

iii.) $\left.\langle\phi, \psi\rangle\right|_{\lambda=z}$ is a holomorphic function on $B_{\hbar}^{-}$and

$$
\left.\langle\phi, \psi\rangle\right|_{\lambda=z}=\left.\left.\sum_{k=1}^{\infty}\left\langle\phi, e_{k}\right\rangle\right|_{\lambda=z}\left\langle e_{k}, \psi\right\rangle\right|_{\lambda=z}
$$

converges normally on $B_{\hbar}^{-}$.

iv.) The quotient space $\mathcal{H}(\hbar):=H(\hbar) / N(\hbar)$ with the induced Hermitian product is a $\mathbb{C}$ Hilbert space with Hilbert base $\left\{e_{k} \bmod N(\hbar)\right\}_{k \in \mathbb{N}}$ and hence $\mathcal{H}(\hbar)$ is isometric to $\ell^{2}(\mathbb{C})$. 
Note that in both cases the resulting $\mathbb{C}$-Hilbert space $\mathcal{H}$ is already a Hilbert space and not only a pre-Hilbert space.

Remark: If one defines $F_{k}$ with $a_{q_{r}}^{(k)}$ instead of its absolute value then one would obtain again two $\mathbb{C}$-subvector spaces and again the quotient would be a Hilbert space. But now $\left.\langle\phi, \psi\rangle\right|_{\lambda=\hbar}$ is in general not convergent to $\langle\phi, \psi\rangle_{\hbar}$ as in equation (59). Consider for example $\phi_{1}:=\sum_{k=0}^{\infty} \lambda^{k} \sin \left(\frac{\pi \lambda}{2}\right) e_{k}$ or $\phi_{2}:=\sum_{r=0}^{\infty} k ! \lambda^{k} \sin \left(\frac{\pi \lambda}{2}\right) e_{k}$ which are both in $H(2)$ but clearly $\left.\left\langle\phi_{1}, \phi_{1}\right\rangle\right|_{\lambda=\hbar}$ has only convergence radius 1 and $\left.\left\langle\phi_{2}, \phi_{2}\right\rangle\right|_{\lambda=\hbar}$ has convergence radius 0 while $\left\langle\phi_{1}, \phi_{1}\right\rangle_{\hbar}=\left\langle\phi_{2}, \phi_{2}\right\rangle_{\hbar}=0$.

Now we want to consider the Fedosov star product of Wick type for a Kähler manifold $M$ of real dimension $2 n$ constructed as in [9]. We will use the same notation as in [9] with the only difference that the formal parameter in denoted by $\lambda$ and $\hbar$ is reserved for the real number corresponding to the value of Planck's constant in a chosen unit system. Let $\omega$ be the symplectic Kähler form on $M$ which is given in local holomorphic coordinates by $\omega=\frac{i}{2} \omega_{k \bar{l}} d z^{k} \wedge d \bar{z}^{l}$. The Fedosov algebra is defined by

$$
\mathcal{W} \otimes \Lambda:=\left(\mathbf{X}_{s=0}^{\infty} \mathbb{C}\left(\Gamma\left(\bigvee^{s} T^{*} M \otimes \bigwedge^{*} M\right)\right)\right)\langle\langle\lambda\rangle\rangle
$$

together with the pointwise multiplication induced by the symmetric and antisymmetric product of forms. The elements $a \in \mathcal{W} \otimes \Lambda$ are of the form $\sum_{r=0}^{\infty} \lambda^{q_{r}} a_{q_{r}}$ where $a_{q_{r}}=\sum_{s=0}^{\infty} a_{s}^{\left(q_{r}\right)}$ and $a_{s}^{\left(q_{r}\right)} \in \mathbb{C}\left(\Gamma\left(\bigvee^{s} T^{*} M \otimes \bigwedge T^{*} M\right)\right)$ are smooth sections. The fibrewise Wick product as a deformation of the pointwise product is defined by

$$
\begin{gathered}
a \circ b:=\sum_{r=0}^{\infty}\left(\frac{i \lambda}{2}\right)^{r} \Lambda^{(r)}(a, b) \\
\Lambda^{(r)}(a, b):=\frac{1}{r !}\left(\frac{4}{i}\right)^{r} \omega^{k_{1} \bar{l}_{1}} \cdots \omega^{k_{r} \bar{l}_{r}} i_{s}\left(Z_{k_{1}}\right) \cdots i_{s}\left(Z_{k_{r}}\right) a i_{s}\left(\bar{Z}_{l_{1}}\right) \cdots i_{s}\left(\bar{Z}_{l_{r}}\right) b .
\end{gathered}
$$

and since this product is defined fibrewise we can define the Fedosov algebra at a point $p \in M$ by

$$
\mathcal{W} \otimes \Lambda_{p}:=\left(\mathrm{X}_{s=0}^{\infty} \mathbb{C}\left(\bigvee^{s} T^{*} M \otimes \bigwedge T^{*} M\right)\right)\langle\langle\lambda\rangle\rangle
$$

together with the product $\circ$. Then the restriction $a_{p}$ of a section $a \in \mathcal{W} \otimes \Lambda$ to the point $p \in M$ is an element in $\mathcal{W} \otimes \Lambda_{p}$. The sections in $\mathcal{W} \otimes \Lambda$ without any antisymmetric part are denoted by $\mathcal{W}$ and analogously we define $\mathcal{W}_{p}$. In [9] we have shown that there exists a Fedosov derivation $D$ for $\mathcal{W} \otimes \Lambda$ such that $D^{2}=0$ and $\mathcal{W}_{D}:=\operatorname{ker} D \cap \mathcal{W}$ is isomorphic to $C^{\infty}(M)\langle\langle\lambda\rangle\rangle$ and the isomorphism is given by the Fedosov-Taylor series $\tau: C^{\infty}(M)\langle\langle\lambda\rangle\rangle \rightarrow \mathcal{W}_{D}$ which was constructed recursively and the inverse map $\sigma: \mathcal{W}_{D} \rightarrow C^{\infty}(M)\langle\langle\lambda\rangle\rangle$ is simply the projection on the elements with symmetric degree zero, i.e. $\sigma=\pi^{(0,0)}$ where $\pi^{(r, s)}$ is the projection onto the symmetric forms of type $(r, s)$. Then the Fedosov star product is given by $f * g=\sigma(\tau(f) \circ \tau(g))$ and several properties of this star product were shown in [9]. The most important property for our purpose is the reality of the Fedosov-Taylor series $\tau(\bar{f})=\overline{\tau(f)}$.

Lemma 6.4 Let $M$ be a 2n-dim. Kähler manifold and $z^{1}, \ldots, z^{n}$ a holomorphic chart around $p \in M$ such that $\left.\omega_{k \bar{l}}\right|_{p}=\delta_{k \bar{l}}$ in this chart. Then $\varphi(1):=1, \varphi\left(d z^{k}\right):=y^{k}, \varphi\left(d \bar{z}^{k}\right):=\bar{y}^{k}$ induces a $\mathbb{C}\langle\langle\lambda\rangle\rangle$-algebra isomorphism $\varphi: \mathcal{W}_{p} \rightarrow \mathcal{W}_{n}$ with respect to the fibrewise Wick product in $\mathcal{W}_{p}$ and the Wick product (55) in $\mathcal{W}_{n}$. 
Note that it is always possible to find such a holomorphic chart [17, Sec. 0.7]. With such an isomorphism we easily find the following proposition analogously to lemma 5.1:

Proposition 6.5 Let $p \in M$ and $\delta_{p}$ the delta functional at $p$. Then $\delta_{p}: C^{\infty}(M)\langle\langle\lambda\rangle \rightarrow$ $\mathbb{C}\langle\langle\lambda\rangle\rangle$ is a state with respect to the Fedosov star product of Wick type and the Gel'fand ideal is given by

$$
\mathcal{J}_{p}:=\left\{f \in C^{\infty}(M)\langle\langle\lambda\rangle\rangle \mid \forall r \geq 0: \pi^{(0, r)} \tau_{p}(f)=0\right\}
$$

where $\tau_{p}(f)$ is the Fedosov-Taylor series of $f$ evaluated at $p$.

In the next proposition we shall prove that the Fedosov-Taylor series $\tau_{p}$ at a point $p \in M$ is surjective on $\mathcal{W}_{p}$ where we use Borel's lemma and the recursion formula [9, eqn. (19)] for $\tau$.

Proposition 6.6 The Fedosov-Taylor series $\tau_{p}: C^{\infty}(M)\langle\langle\lambda\rangle\rangle \rightarrow \mathcal{W}_{p}$ is surjective for all $p \in M$.

We define for $p \in M$

$$
\widetilde{\mathcal{J}}_{p}:=\left\{a \in \mathcal{W}_{p} \mid \forall r \geq 0: \pi^{(0, r)} a=0\right\} \quad{\widetilde{\mathfrak{H}^{\prime}}}_{p}^{\prime}:=\left(\mathrm{X}_{s=0}^{\infty} \bigvee^{(0, s)} T^{*} M_{p}\right)\langle\langle\lambda\rangle\rangle
$$

and then we can describe the GNS representation induced by $\delta_{p}$ in the following way:

Theorem 6.7 With the notations from above we have:

i.) The quotient space $\mathfrak{H}_{p}^{\prime}:=C^{\infty}(M)\langle\langle\lambda\rangle\rangle / \mathcal{J}_{p}$ is canonically isomorphic to $\widetilde{\mathfrak{H}}_{p}^{\prime}$ where the isomorphism is induced by $\tau_{p}$ (and also denoted by $\tau_{p}$ ):

$$
\psi_{f} \mapsto \tau_{p}\left(\psi_{f}\right):=\tau_{p}(f) \bmod \widetilde{\mathcal{J}}_{p}=\sum_{r=0}^{\infty} \frac{1}{r !}\left(\pi^{(0,0)} i_{s}\left(\bar{Z}_{k_{1}}\right) \cdots i_{s}\left(\bar{Z}_{k_{r}}\right) \tau_{p}(f)\right) d \bar{z}^{k_{1}} \vee \cdots \vee d \bar{z}^{k_{r}}
$$

where $\psi_{f} \in \mathfrak{H}_{p}^{\prime}$ and the Hermitian product is given by

$$
\left\langle\psi_{f}, \psi_{g}\right\rangle=\sum_{r=0}^{\infty} \frac{(2 \lambda)^{r}}{r !} \omega_{p}^{k_{1} \bar{l}_{1}} \cdots \omega_{p}^{k_{r} \bar{l}_{r}} \pi^{(0,0)}\left(i_{s}\left(Z_{k_{1}}\right) \cdots i_{s}\left(Z_{k_{r}}\right) \overline{\tau_{p}(f)} i_{s}\left(\bar{Z}_{l_{1}}\right) \cdots i_{s}\left(\bar{Z}_{l_{r}}\right) \tau_{p}(g)\right)
$$

and the GNS representation induced by $\delta_{p}$ is given by

$$
\begin{aligned}
\tau_{p} \circ \pi_{p}(f) \circ \tau_{p}^{-1}= & \sum_{r, s=0}^{\infty} \frac{(2 \lambda)^{r}}{r ! s !}\left(\pi^{(0,0)}\left(i_{s}\left(Z_{k_{1}}\right) \cdots i_{s}\left(Z_{k_{r}}\right) i_{s}\left(\bar{Z}_{l_{1}}\right) \cdots i_{s}\left(Z_{l_{s}}\right) \tau_{p}(f)\right)\right) \\
& \times \omega^{k_{1} \bar{t}_{1}} \cdots \omega^{k_{r} \bar{t}_{r}} d \bar{z}^{l_{1}} \vee \cdots \vee d \bar{z}^{l_{s}} i_{s}\left(\bar{Z}_{t_{1}}\right) \cdots i_{s}\left(\bar{Z}_{t_{r}}\right)
\end{aligned}
$$

ii.) Let $z^{1}, \ldots, z^{n}$ be a holomorphic chart such that $\left.\omega_{k \bar{l}}\right|_{p}=\delta_{k \bar{l}}$ and let $\varphi: \mathcal{W}_{p} \rightarrow \mathcal{W}_{n}$ be defined as in lemma 6.4. Then $\varphi \circ \tau_{p}$ induces an isomorphism (also denoted by $\varphi \circ \tau_{p}$ ) of the $\mathbb{C}\left\langle\langle\lambda\rangle\right.$-pre-Hilbert space $\mathfrak{H}_{p}^{\prime}$ to $\mathfrak{H}_{n}^{\prime}$ and hence the completion $\mathfrak{H}_{p}$ of $\mathfrak{H}_{p}^{\prime}$ is isometric to $\mathfrak{H}_{n}$ via $\varphi \circ \tau_{p}$ and hence $\mathfrak{H}_{p}$ has a countable Hilbert base and is isometric to $\ell^{2}(\mathbb{C}\langle\langle\lambda\rangle\rangle)$. Moreover we have for $\psi_{f} \in \mathfrak{H}_{p}^{\prime}$

$$
\varphi \circ \tau_{p}\left(\psi_{f}\right)=\left.\sum_{r=0}^{\infty} \frac{1}{r !} \frac{\partial^{r} \varphi \circ \tau_{p}(f)}{\partial \bar{y}^{k_{1}} \cdots \partial \bar{y}^{k_{r}}}\right|_{y=0} \bar{y}^{k_{1}} \cdots \bar{y}^{k_{r}}
$$


As in the Wick case we can ask for convergence. First we have to choose a Hilbert base of $\mathfrak{H}_{p}$. In a holomorphic chart $z^{1}, \ldots, z^{n}$ with $\left.\omega_{k \bar{l}}\right|_{p}=\delta_{k \bar{l}}$ we can use for example the vectors $\hat{e}_{K}:=\left(\varphi \circ \tau_{p}\right)^{-1} e_{K}$ with $e_{K}$ as in proposition 6.1. Then we construct $H_{p}(\hbar)$ and $N_{p}(\hbar)$ with respect to this Hilbert base as in theorem 6.3 and get a $\mathbb{C}$-Hilbert space $\mathcal{H}_{p}(\hbar)$ and a representation of 'many' functions $f \in C^{\infty}(M)\langle\langle\lambda\rangle\rangle$ in the same way as in the Wick case. We consider again functions $f$ with $\pi_{p}(f) N_{p}(\hbar) \subseteq N_{p}(\hbar)$ and define $D_{f}(\hbar)$ and $\mathcal{D}_{f}(\hbar)$ as in the Wick case. Then we define the quantization map $\mathcal{Q}$ analogously: $\mathcal{Q}(f)$ is an operator on $\mathcal{D}_{f}(\hbar)$ defined by

$$
\mathcal{Q}(f)\left(\psi \bmod N_{p}(\hbar)\right):=\pi_{p}(f) \psi \bmod N_{p}(\hbar) \quad \psi \in D_{f}(\hbar)
$$

which leads to the representation property $\mathcal{Q}(f) \circ \mathcal{Q}(g)=\mathcal{Q}(f * g)$ on suitable domains. Since according to proposition 6.6 the Fedosov-Taylor series is surjective there are indeed 'many' suitable functions such as the pre-images under $\tau_{p}$ of the polynomials in $d z^{k}$ and $d \bar{z}^{k}$ (where $z^{1}, \ldots, z^{n}$ is the chart from above).

\section{Example II: The Weyl-Moyal product for $\mathbb{R}^{2 n}$}

Now we want to consider the phase space $T^{*} \mathbb{R}^{n} \cong \mathbb{R}^{2 n}$ with the standard symplectic form and the Weyl-Moyal product defined for $f, g \in C^{\infty}\left(\mathbb{R}^{2 n}\right)\langle\langle\lambda\rangle\rangle$ by

$$
\begin{gathered}
f * g:=\sum_{r=0}^{\infty}\left(\frac{i \lambda}{2}\right)^{r} \Lambda^{(r)}(f, g) \\
\Lambda^{(r)}(f, g):=\frac{1}{r !} \Lambda^{i_{1} j_{1}} \cdots \Lambda^{i_{r} j_{r}} \frac{\partial^{r} f}{\partial x^{i_{1}} \cdots \partial x^{i_{r}}} \frac{\partial^{r} g}{\partial x^{j_{1}} \cdots \partial x^{j_{r}}}
\end{gathered}
$$

where $x^{1}, \ldots, x^{2 n}=q^{1}, \ldots, q^{n}, p_{1}, \ldots p_{n}$ and $\Lambda^{i j}$ are the components of the Poisson tensor $\Lambda$ with respect to the coordinates $x^{1}, \ldots, x^{2 n}$, i.e. $\Lambda=\frac{1}{2} \Lambda^{i j} \partial_{x^{i}} \wedge \partial_{x^{j}}=\partial_{q^{i}} \wedge \partial_{p_{i}}$. The smooth functions on $\mathbb{R}^{2 n}$ with compact support are denoted by $C_{0}^{\infty}\left(\mathbb{R}^{2 n}\right)\langle\langle\lambda\rangle\rangle$.

Lemma 7.1 Let $f, g \in C_{0}^{\infty}\left(\mathbb{R}^{2 n}\right)\langle\langle\lambda\rangle\rangle$ then the integral over $\mathbb{R}^{2 n}$ is a trace 19

$$
\int_{\mathbb{R}^{2 n}} f * g d^{2 n} x=\int_{\mathbb{R}^{2 n}} g * f d^{2 n} x=\int_{\mathbb{R}^{2 n}} f g d^{2 n} x
$$

and clearly a positive linear functional.

To obtain Schrödinger's quantization we need a different positive linear functional namely the integration over the configuration space $Q:=\mathbb{R}^{n}$ of $T^{*} Q \cong \mathbb{R}^{2 n}$ for a fixed value $\overrightarrow{p_{0}}$ of the momenta. But first we have to define a suitable subalgebra of $C^{\infty}\left(T^{*} Q\right)\langle\langle\lambda\rangle\rangle$ such that the integration is well-defined. For $\overrightarrow{p_{0}} \in \mathbb{R}^{n}$ we define

$$
C_{\overrightarrow{p_{0}}}^{\infty}\left(T^{*} Q\right):=\left\{f \in C^{\infty}\left(T^{*} Q\right) \mid \operatorname{supp} f \cap Q_{\overrightarrow{p_{0}}} \text { is compact }\right\}
$$

where $Q_{\overrightarrow{p_{0}}}:=\left\{\left(\vec{q}, \overrightarrow{p_{0}}\right) \in T^{*} Q \mid \vec{q} \in Q\right\}$ and notice that due to the locality of the Weyl-Moyal product $C_{\overrightarrow{p_{0}}}^{\infty}\left(T^{*} Q\right)\langle\langle\lambda\rangle\rangle$ is not only a subalgebra but also a twosided ideal of $C^{\infty}\left(T^{*} Q\right)\langle\langle\lambda\rangle\rangle$. 
As we will see in proposition 7.4 it is not sufficient to consider only the functions with compact support in $T^{*} Q$. For $f \in C_{\overrightarrow{p_{0}}}^{\infty}\left(T^{*} Q\right)\langle\langle\lambda\rangle\rangle$ we define

$$
\omega_{\overrightarrow{p_{0}}}(f):=\int_{\mathbb{R}^{n}} f\left(\vec{q}, \overrightarrow{p_{0}}\right) d^{n} q
$$

which is obviously well-defined for any fixed momentum $\overrightarrow{p_{0}}$. Moreover it is a positive linear functional of $C_{\overrightarrow{p_{0}}}^{\infty}\left(T^{*} Q\right)\langle\langle\lambda\rangle\rangle$ :

Proposition 7.2 Let $f, g \in C_{\overrightarrow{p_{0}}}^{\infty}\left(T^{*} Q\right)\langle\langle\lambda\rangle\rangle$ and define $\Delta:=\frac{\partial^{2}}{\partial q^{k} \partial p_{k}}$ and $S:=\exp \left(-\frac{i \lambda}{2} \Delta\right)$ then

$$
\omega_{\overrightarrow{p_{0}}}(f * g)=\left.\int_{\mathbb{R}^{n}}\left(\left(S^{-1} f\right)(S g)\right)\right|_{\vec{p}=\overrightarrow{p_{0}}} d^{n} q
$$

and $\omega_{\overrightarrow{p_{0}}}$ is positive, i.e. $\omega_{\overrightarrow{p_{0}}}(\bar{f} * f) \geq 0$.

PROOF: A straight forward induction on $r$ shows that

$$
\left.r ! \int_{\mathbb{R}^{n}} \Lambda^{r}(f, g)\right|_{\vec{p}=\overrightarrow{p_{0}}} d^{n} q=\left.\int_{\mathbb{R}^{n}}\left(\sum_{s=0}^{r}\left(\begin{array}{l}
r \\
s
\end{array}\right) \Delta^{s} f(-\Delta)^{r-s} g\right)\right|_{\vec{p}=\overrightarrow{p_{0}}} d^{n} q
$$

then equation (67) follows easily and this clearly implies the positivity.

Remark: A similar but non-formal positivity result of such integrals was obtained in [14, pp. 205-206 eqn. 5.11] in the context of complex $C^{*}$-algebras.

Now we shall specialize to $\overrightarrow{p_{0}}=0$ for simplicity. We define for $k=1, \ldots, n$ the functions $P_{k}(\vec{q}, \vec{p}):=p_{k}$ and denote by $\mathcal{J}_{0}$ the left ideal of $C_{Q}^{\infty}\left(T^{*} Q\right)\langle\langle\lambda\rangle\rangle:=C_{\overrightarrow{p_{0}}=0}^{\infty}\left(T^{*} Q\right)\langle\langle\lambda\rangle\rangle$ generated by the functions $P_{k}$

$$
\mathcal{J}_{0}:=\left\{f \in C^{\infty}\left(T^{*} Q\right)\langle\langle\lambda\rangle\rangle \mid f=\sum_{k} g_{k} * P_{k}, g_{k} \in C_{Q}^{\infty}\left(T^{*} Q\right)\langle\langle\lambda\rangle\rangle\right\}
$$

which is indeed a left ideal of $C_{Q}^{\infty}\left(T^{*} Q\right)\langle\langle\lambda\rangle\rangle$ since $C_{Q}^{\infty}\left(T^{*} Q\right)\langle\langle\lambda\rangle\rangle$ is a twosided ideal of the whole algebra $C^{\infty}\left(T^{*} Q\right)\langle\langle\lambda\rangle\rangle$. By direct calculation we get:

Lemma 7.3 Let $f_{1}, \ldots, f_{n} \in C_{Q}^{\infty}\left(T^{*} Q\right)\langle\langle\lambda\rangle\rangle$ then we have

$$
\omega_{0}\left(\overline{\left(f_{k} * P_{k}\right)} *\left(f_{l} * P_{l}\right)\right)=0
$$

and $\mathcal{J}_{0}$ is contained in the Gel'fand ideal of $\omega_{0}$.

The next proposition will show that $\mathcal{J}_{0}$ is already equal to the Gel'fand ideal of $\omega_{0}$ and the quotient $\mathfrak{H}_{0}:=C_{Q}^{\infty}\left(T^{*} Q\right)\langle\langle\lambda\rangle\rangle / \mathcal{J}_{0}$ is canonically isomorphic to $C_{0}^{\infty}(Q)\langle\langle\lambda\rangle\rangle$. Let $r_{0}$ : $C^{\infty}\left(T^{*} Q\right)\langle\langle\lambda\rangle\rangle \rightarrow C^{\infty}(Q)\langle\langle\lambda\rangle\rangle$ be the restriction to momentum $\vec{p}=0$, i.e. $r_{0}(f)(\vec{q}):=f(\vec{q}, 0)$ and let $\pi_{Q}: T^{*} Q \rightarrow Q$ be the projection to the configuration space. Then we clearly have $r_{0}\left(C_{Q}^{\infty}\left(T^{*} Q\right)\langle\langle\lambda\rangle\rangle\right)=C_{0}^{\infty}(Q)\langle\langle\lambda\rangle\rangle$ and we define $i_{0}:=\pi_{Q}^{*} \circ r_{0}$, i.e. $i_{0}(f)(\vec{q}, \vec{p}):=f(\vec{q}, 0)$. Moreover we need the operator $I: C_{Q}^{\infty}\left(T^{*} Q\right)\langle\langle\lambda\rangle\rangle \rightarrow C_{Q}^{\infty}\left(T^{*} Q\right)\langle\langle\lambda\rangle\rangle$ defined for $f \in C_{Q}^{\infty}\left(T^{*} Q\right)\langle\langle\lambda\rangle\rangle$ by

$$
I(f)(\vec{q}, \vec{p}):=\int_{0}^{1} \frac{f(\vec{q}, t \vec{p})-f(\vec{q}, 0)}{t} d t
$$


and for $k=1, \ldots, n$ we define $T^{k}: C_{Q}^{\infty}\left(T^{*} Q\right)\langle\langle\lambda\rangle\rangle \rightarrow C_{Q}^{\infty}\left(T^{*} Q\right)\langle\langle\lambda\rangle\rangle$ by

$$
T^{k}(f):=\frac{\partial}{\partial p_{k}}\left(I \circ \frac{1}{1+\frac{i \lambda}{2} \Delta \circ I}(f)\right) .
$$

Note that $I(f), T^{k}(f) \in C_{Q}^{\infty}\left(T^{*} Q\right)\langle\langle\lambda\rangle\rangle$ if $f \in C_{Q}^{\infty}\left(T^{*} Q\right)\langle\langle\lambda\rangle\rangle$.

Proposition 7.4 With the above notation we have for $f \in C_{Q}^{\infty}\left(T^{*} Q\right)\langle\langle\lambda\rangle\rangle$ :

i.) $f=i_{0} \circ S(f)+T^{k}(f) * P_{k}$

ii.) This decomposition is unique, i.e.

$$
C_{Q}^{\infty}\left(T^{*} Q\right)\langle\langle\lambda\rangle\rangle \cong i_{0} \circ S\left(C_{Q}^{\infty}\left(T^{*} Q\right)\langle\langle\lambda\rangle\rangle\right) \oplus \mathcal{J}_{0}
$$

iii.) The Gel'fand ideal of $\omega_{0}$ is given by $\mathcal{J}_{0}$.

ProOF: First we use Hadamard's trick to obtain $f=i_{0}(f)+P_{k} \partial_{p_{k}} I(f)$ which could be rewritten as $f=i_{0}(f)+\left(\partial_{p_{k}} I(f)\right) * P_{k}-\frac{i \lambda}{2} \Delta \circ I(f)$ using the Weyl-Moyal product. Iterating this equation leads to $f=i_{0} \circ \frac{1}{1+\frac{i \lambda}{2} \Delta \circ I}(f)+T^{k}(f) * P_{k}$. An easy induction shows that $r ! i_{0} \circ(\Delta \circ I)^{r}=i_{0} \circ \Delta^{r}$ which proves the first part. The second part follows from lemma 7.3 , and the last statement is a consequence of part one and two.

Corollary 7.5 The quotient space $\mathfrak{H}_{0}:=C_{Q}^{\infty}\left(T^{*} Q\right)\langle\langle\lambda\rangle\rangle / \mathcal{J}_{0}$ with the Hermitian product $\langle\cdot, \cdot\rangle$ induced by $\omega_{0}$ is canonically isometric to $C_{0}^{\infty}(Q)\langle\langle\lambda\rangle\rangle$ with the Hermitian product

$$
\langle\psi, \phi\rangle:=\int_{\mathbb{R}^{n}} \overline{\psi(\vec{q})} \phi(\vec{q}) d^{n} q \quad \psi, \phi \in C_{0}^{\infty}(Q)\langle\langle\lambda\rangle\rangle
$$

and the canonical isometry $r$ is induced by $r_{0}:$ For $\psi_{f} \in \mathfrak{H}_{0}$ we set $r\left(\psi_{f}\right):=r_{0} \circ S(f)$ and the inverse of $r$ is simply the pull-back $\pi_{Q}^{*} \bmod \mathcal{J}_{0}$.

Now we consider the GNS representation $\pi_{0}$ induced by $\omega_{0}$ on $\mathfrak{H}_{0}$. First we notice that according to corollary 3.28 not only $C_{Q}^{\infty}\left(T^{*} Q\right)\langle\langle\lambda\rangle\rangle$ could be represented but the whole algebra $C^{\infty}\left(T^{*} Q\right)\langle\langle\lambda\rangle\rangle$ since $C_{Q}^{\infty}\left(T^{*} Q\right)\langle\langle\lambda\rangle\rangle$ is a twosided ideal and $\mathcal{J}_{0}$ is a left ideal of $C^{\infty}\left(T^{*} Q\right)\langle\langle\lambda\rangle\rangle$. Let $\varrho$ be the corresponding representation on $C_{0}^{\infty}(Q)\langle\langle\lambda\rangle\rangle$, i.e. $\varrho(f):=r \circ \pi_{0}(f) \circ \pi_{Q}^{*}$.

Theorem 7.6 (Formal Schrödinger Quantization) Let $Q=\mathbb{R}^{n}$ and $\psi \in C_{0}^{\infty}(Q)\langle\langle\lambda\rangle\rangle$ be a 'formal wave function' on the configuration space $Q$ and $f \in C^{\infty}\left(T^{*} Q\right)\langle\langle\lambda\rangle\rangle$.

i.)

$$
\varrho(f) \psi=\left.\sum_{r=0}^{\infty} \frac{1}{r !}\left(\frac{\lambda}{i}\right)^{r} \frac{\partial^{r}(S f)}{\partial p_{i_{1}} \cdots \partial p_{i_{r}}}\right|_{\vec{p}=0} \frac{\partial^{r} \psi}{\partial q^{i_{1}} \cdots \partial q^{i_{r}}}
$$

ii.) For polynomials in $q^{1}, \ldots, p_{n}$ the representation $\varrho$ is the canonical quantization rule, i.e.

$$
\varrho\left(q^{k}\right)=q^{k} \quad \varrho\left(p_{k}\right)=\frac{\lambda}{i} \frac{\partial}{\partial q^{k}}
$$

and the polynomials are mapped to the Weyl ordered polynomials of the corresponding operators (symmetrization rule). 
iii.) The $\mathbb{C}\langle\langle\lambda\rangle\rangle$-pre-Hilbert space $C_{0}^{\infty}(Q)\langle\langle\lambda\rangle\rangle$ with the Hermitian product (73) is already Cauchy complete and hence a $\mathbb{C}\langle\langle\lambda\rangle\rangle$-Hilbert space.

iv.) $\mathfrak{H}_{0}$ does not admit a (countable or uncountable) Hilbert base (for $n \geq 1$ ).

ProOF: The first statement is a straight-forward computation. For the second one notes first that for each nonnegative integer $k$ and $2 n$ formal parameters $\alpha_{1}, \ldots, \alpha_{n}, \beta^{1}, \ldots, \beta^{n}$ the function $\left(\alpha_{r} q^{r}+\beta^{r} p_{r}\right)^{k}$ is assigned the operator $\left(\alpha_{r} \varrho\left(q^{r}\right)+\beta^{r} \varrho\left(p_{r}\right)\right)^{k}$ by the Weyl symmetrization rule. Hence the formal exponential function $e_{\alpha, \beta}(\vec{q}, \vec{p}):=\exp \left(\alpha_{r} q^{r}+\beta^{r} p_{r}\right)$ is assigned the operator $\exp \left(\alpha_{r} \varrho\left(q^{r}\right)+\beta^{r} \varrho\left(p_{r}\right)\right)$. On the other hand, the right hand side of (74) is easily seen to be equal to the standard ordering prescription of the function $S f$, i.e. where after appplying the rule (75) all the derivatives are put on the right hand side first. Clearly, the function $S e_{\alpha, \beta}=\exp (i \lambda \alpha \beta / 2) e_{\alpha, \beta}$ is assigned the operator $\exp (i \lambda \alpha \beta / 2) \exp \left(\alpha_{i} \varrho\left(q^{i}\right)\right) \exp \left(\beta^{i} \varrho\left(p_{i}\right)\right)$ by standard ordering. Now the Baker-Campbell-Hausdorff formula for the $2 n+1$-dimensional Heisenberg Lie algebra easily implies that the Weyl ordered operator for $e_{\alpha, \beta}$ and the standard-ordered operator for $S e_{\alpha, \beta}$ coincide which proves this statement. The third and fourth statement are proved in a more general context in the next theorem.

Theorem 7.7 Let $M$ be an orientable manifold with volume form $\Omega$. Then $\mathfrak{H}:=C_{0}^{\infty}(M)\langle\langle\lambda\rangle\rangle$ together with the integral

$$
\langle f, g\rangle:=\int_{M} \bar{f} g \Omega \quad f, g \in \mathfrak{H}
$$

as Hermitian product is a $\mathbb{C}\langle\langle\lambda\rangle\rangle$-Hilbert space. Moreover $\mathfrak{H}$ does not admit a (countable or uncountable) Hilbert base if $\operatorname{dim} M \geq 1$.

ProOF: It is easy to see that every Cauchy sequence in $\mathfrak{H}$ with respect to the norm induced by the scalar product is a Cauchy sequence in the metric sense of Proposition 4.3, and vice versa, which proves completeness.

Suppose there would exist a Hilbert base $\left\{\psi^{(\alpha)}\right\}_{\alpha \in I}, I$ some index set, for $\mathfrak{H}$. Because of its orthonormality every element is of the form $\psi^{(\alpha)}=\psi_{0}^{(\alpha)}+\lambda^{a_{\alpha}} \psi_{1}^{(\alpha)}$ where the $\psi_{0}^{(\alpha)}$ is an orthonormal family in $C_{0}^{\infty}(M), a_{\alpha}$ is a positive rational number, and $\psi_{1}^{(\alpha)} \in C_{0}^{\infty}(M)\langle\langle\lambda\rangle\rangle$ with vanishing coefficients of neagative $\lambda$-powers. By the usual complex $L^{2}$-theory for manifolds of dimension greater or equal than 1 there are countably many different $\psi_{0}^{(\alpha)}$, and there is a function $f \in C_{0}^{\infty}(M)$ which is not a finite linear combination of the $\psi_{0}^{(\alpha)}$. If $f$ were approximated by finite sums of the $\psi^{(\alpha)}$ there would be a positive integer $N$ and $\alpha_{1}, \ldots, \alpha_{N} \in$ $I$ such that

$$
\lambda^{2}>\left\|f-\sum_{i=1}^{N}\left\langle\psi^{\left(\alpha_{i}\right)}, f\right\rangle\right\|^{2}=\langle f, f\rangle-\sum_{i=1}^{N}\left\langle f, \psi^{\left(\alpha_{i}\right)}\right\rangle\left\langle\psi^{\left(\alpha_{i}\right)}, f\right\rangle
$$

In particular it would follow that $\langle f, f\rangle=\sum_{i=1}^{N}\left\langle f, \psi_{0}^{\left(\alpha_{i}\right)}\right\rangle\left\langle\psi_{0}^{\left(\alpha_{i}\right)}, f\right\rangle$ implying by Parseval's equality for complex Hilbert spaces that $f$ is a finite linear combination of the $\psi^{\left(\alpha_{i}\right)}$ which is a contradiction.

At last we want to describe again the way back to convergence if we substitute the formal parameter $\lambda$ by $\hbar \in \mathbb{R}^{+}$and again we want to ask for convergence in the representation. Let 
$\psi=\sum_{r=0}^{\infty} \lambda^{q_{r}} \psi_{q_{r}} \in C_{0}^{\infty}(Q)\langle\langle\lambda\rangle\rangle$ be a formal wave function. Then we define its Fourier transform order by order

$$
\widetilde{\psi}(\vec{k}):=\sum_{r=0}^{\infty} \lambda^{q_{r}} \widetilde{\psi}_{q_{r}}(\vec{k}) \quad \text { where } \quad \widetilde{\psi}_{q_{r}}(\vec{k}):=\frac{1}{(2 \pi)^{\frac{n}{2}}} \int_{Q} \psi_{q_{r}}(\vec{q}) e^{i \vec{k} \cdot \vec{q}} d^{n} q \quad \vec{k} \in \mathbb{R}^{n} \text {. }
$$

Since $\psi \in C_{0}^{\infty}\left(\mathbb{R}^{n}\right)\langle\langle\lambda\rangle\rangle$ we have $\widetilde{\psi} \in \mathcal{S}\left(\mathbb{R}^{n}\right)\langle\langle\lambda\rangle\rangle$ where $\mathcal{S}\left(\mathbb{R}^{n}\right)$ is Schwarz's space of rapidly decreasing smooth functions. Moreover we define the truncated series $\psi_{N}:=\sum_{r=0}^{N} \lambda^{q_{r}} \psi_{q_{r}}$. Then we ask for $L^{2}$-convergence of the sequence $\widetilde{\psi}_{N}$ if we substitute $\lambda$ by $\hbar$ and define

$$
\begin{aligned}
& H(\hbar):=\left\{\psi \in C_{0}^{\infty}\left(\mathbb{R}^{n}\right)\langle\langle\lambda\rangle\rangle\left|\widetilde{\psi_{N}}\right|_{\lambda=\hbar} \stackrel{L^{2}}{\longrightarrow} \widetilde{\Psi} \in L^{2}\left(\mathbb{R}^{n}\right) \text { as } N \rightarrow \infty\right\} \\
& N(\hbar):=\left\{\psi \in H(\hbar)\left|\widetilde{\psi_{N}}\right|_{\lambda=\hbar} \stackrel{L^{2}}{\longrightarrow} 0 \text { as } N \rightarrow \infty\right\} .
\end{aligned}
$$

Now the same procedure as in the Wick case could be done and we get:

Theorem 7.8 With the notations of above we have:

i.) $H(\hbar)$ is a $\mathbb{C}$-vector space and $N(\hbar)$ is a $\mathbb{C}$-subvector space of $H(\hbar)$.

ii.) $\psi \in H(\hbar)$ iff $\left.\psi_{N}\right|_{\lambda=\hbar} L^{2}$-converges to some $\Psi \in L^{2}\left(\mathbb{R}^{n}\right)$. Then $\widetilde{\Psi}$ is the Fourier transform of $\Psi$.

iii.) If $\psi, \phi \in H(\hbar)$ then $\langle\psi, \phi\rangle$ converges for $\lambda=\hbar$ and

$$
\left.\langle\psi, \phi\rangle\right|_{\lambda=\hbar}=\int_{\mathbb{R}^{n}} \overline{\Psi(\vec{q})} \Phi(\vec{q}) d^{n} q=\int_{\mathbb{R}^{n}} \overline{\widetilde{\Psi}(\vec{q})} \widetilde{\Phi}(\vec{q}) d^{n} q
$$

and defines a positive semidefinite sesquilinear form for $H(\hbar)$.

iv.) The quotient $\mathcal{H}(\hbar):=H(\hbar) / N(\hbar)$ is canonically isometric to the $\mathbb{C}$-Hilbert space $L^{2}\left(\mathbb{R}^{n}\right)$ where $\psi \bmod N(\hbar) \mapsto \Psi$ is the isomorphism.

Again the GNS representation $\varrho$ induces a representation of at least 'many' elements $f \in$ $C^{\infty}\left(\mathbb{R}^{2 n}\right)\langle\langle\lambda\rangle\rangle$ on $\mathcal{H}(\hbar) \cong L^{2}\left(\mathbb{R}^{n}\right)$. For $f \in C^{\infty}\left(\mathbb{R}^{2 n}\right)\langle\langle\lambda\rangle\rangle$ such that $\varrho(f) N(\hbar) \subseteq N(\hbar)$ we define again $D_{f}(\hbar)$ and $\mathcal{D}_{f}(\hbar)$ as in the Wick case and obtain a quantization map $\mathcal{Q}$ defined by

$$
\mathcal{Q}(f)(\psi \bmod N(\hbar)):=\varrho(f) \psi \bmod N(\hbar) .
$$

for $\psi \in D_{f}(\hbar)$. Then $\mathcal{Q}(f)$ is a linear operator defined on some domain $\mathcal{D}_{f}(\hbar) \subseteq \mathcal{H}(\hbar) \cong$ $L^{2}\left(\mathbb{R}^{n}\right)$ and again there are 'many' elements $f$ with dense domain $\mathcal{D}_{f}(\hbar)$.

\section{Open Problems}

In this section we list some open problems arising with our approach: 
- It would be very interesting to see whether the approach of the WKB approximation via Lagrangean submanifolds (see e. g. [3]) contained in the energy surface of a fixed Hamiltonian function on the symplectic manifold is related to some variant of GNS construction with respect to a suitable positive linear functional whose support is contained in that Lagrangean submanifold. One of the problems arising here is the fact that the discrete energy eigenvalues in quantum mechanics are dependent on $\hbar$, and it is not obvious how this can be encoded in the energy surface.

- We have seen in section 5 that the spectrum of the harmonic oscillator can be computed in a purely formal manner before the convergence scheme is performed. It may be interesting to develop a kind of 'formal spectral theory' in order to formally compute e.g. discrete spectra depending on $\lambda$ and then deal with the convergence.

- Related to this question one may ask more generally to what extend there is some reasonable functional analysis in these formal Hilbert spaces: i.e. which (possibly weaker) topologies are more suited for the definition and calculation of spectra and convergence properties. The usual literature on $p$-adic functional analysis (see e.g. [25]) does unfortunately not seem to deal with Hilbert spaces, but exclusively with absolute values, and seems to avoid ordered fields and hence the notion of positivity we need.

- Finally, it may be interesting to see whether the prequantum line bundles of geometric quantization over, say, a compact prequantizable Kähler manifold (see e.g. [28], [10], [11) are related to this construction (perhaps after employing a convergence scheme) and if yes, whether they can be constructed that way.

\section{Acknowledgements}

The authors would like to thank K. Fredenhagen for asking the question of the GNS construction in deformation quantization and suggesting to us to concentrate on the algebraic properties of $C^{*}$-algebras and O. Kegel for pointing out references [19], [18], and [24]. Furthermore, we would like to thank M. Flato, K. Fredenhagen, J. Huebschmann, H. Rehren, and A. Weinstein for valuable discussions, and J. Hoppe and M. Lledo for a charming tablefootball match.

\section{References}

[1] Basart, H., Flato, M., Lichnerowicz, A., Sternheimer, D.: Deformation Theory applied to Quantization and Statistical Mechanics Lett. Math. Phys. 8, 483-494 (1984).

[2] Basart, H., Lichnerowicz, A.: Conformal Symplectic Geometry, Deformations, Rigidity and Geometrical (KMS) Conditions Lett. Math. Phys. 10, 167-177 (1985).

[3] Bates, S., Weinstein, A.: Lectures on the Geometry of Quantization Berkeley Mathematics Lecture Notes Vol. 8 (1995). 
[4] Bayen, F., Flato, M., Fronsdal, C., Lichnerowicz, A., Sternheimer, D.: Deformation Theory and Quantization. Annals of Physics 111, part I: 61-110, part II: 111-151 (1978).

[5] Bratteli, O., Robinson, D. W.: Operator Algebras and Quantum Statistical Mechanics 1, second edition. (Springer Verlag, New York, Berlin, Heidelberg 1987).

[6] Berezin, F.: Quantization. Izv.Mat.NAUK 38, 1109-1165 (1974).

[7] Bordemann, M., Brischle, M., Emmrich, C., Waldmann, S.: Phase Space Reduction for Star-Products: An Explicit Construction for $\mathbb{C} P^{n}$ Lett. Math. Phys. 36, 357-371 (1996).

[8] Bordemann, M., Brischle, M., Emmrich, C., Waldmann, S.: Subalgebras with Converging Star Products in Deformation Quantization: An Algebraic Construction for $\mathbb{C} P^{n}$. Uni Freiburg Preprint FR-THEP-95/28, December 1995, q-alg/9512019.

[9] Bordemann, M., Waldmann, S.: A Fedosov Star Product of Wick Type for Kähler manifolds FR-THEP-96/9 preprint (1996).

[10] Cahen, M., Gutt, S., Rawnsley, J.: Quantization of Kähler Manifolds I. J. of Geometry and Physics 7, 45-62 (1990).

[11] Cahen, M., Gutt, S., Rawnsley, J.: Quantization of Kähler Manifolds. II. Trans.Am.Math.Soc 337, 73-98 (1993).

[12] Connes, A., Flato, M., Sternheimer, D.: Closed Star Products and Cyclic Cohomology Lett. Math. Phys. 24, 1-12 (1992).

[13] DeWilde, M., Lecomte, P. B. A.: Existence of star-products and of formal deformations of the Poisson Lie Algebra of arbitrary symplectic manifolds. Lett. Math. Phys. 7, 487-496 (1983).

[14] Doplicher, S., Fredenhagen, K., Roberts, J. E.: The Quantum Structure of Spacetime at the Planck Scale and Quantum Fields. Comm. Math. Phys. 172, 187-220 (1995)

[15] Fedosov, B.: A Simple Geometrical Construction of Deformation Quantization. J. of Diff. Geom. 40, 213-238 (1994).

[16] Fedosov, B.: Deformation Quantization and Index Theory. Akademie Verlag, Berlin 1996.

[17] Griffiths, P., Harris, J.: Principles of Algebraic Geometry. John Wiley, New York (1978).

[18] Gross, H. Quadratic Forms in Infinite Dimensional Vector Spaces. Birkhäuser, Boston, Basel, Stuttgart, 1979. 
[19] Gross, H., Keller, A.: On the Definition of Hilbert Space. manuscripta math. 23, 67-90 (1977).

[20] HaAg, R.: Local Quantum Physics, second edition. Springer, Berlin, 1993.

[21] Jacobson, N.: Basic Algebra I second ed. Freeman and Co., New York (1985).

[22] Jacobson, N.: Basic Algebra II second ed. Freeman and Co., New York (1985).

[23] Kelley, J. L.: General Topology. GTM 27, Springer, New York (Reprint of the 1955 edition).

[24] Keller, H. A.: Ein nicht-klassischer Hilbertscher Raum. Math. Z. 172, 41-49 (1980).

[25] Narici, N., Beckenstein, E., Bachman, G.: Functional Analysis and Valuation Theory Marcel Dekker, New York, (1971).

[26] Omori, H., Maeda, Y., Yoshioka, A.: Weyl manifolds and deformation quantization Adv. in Math. 85, 224-255 (1991).

[27] Pflaum, M. J.: Local Analysis of Deformation Quantization. Ph.D. thesis, Fakultät für Mathematik der Ludwig-Maximilians-Universität, München, 1995.

[28] Rawnsley, J.: Coherent States and Kähler Manifolds. Quart. J. Oxford (2), 28, 403-415 (1977).

[29] Rubio, R.: Algèbres associatives locales sur l'espace des sections d'un fibré en droites C.R.A.S. t.299, Série I, 699-701 (1984).

[30] Rudin, W.: Real and Complex Analysis. $3^{\text {rd }}$ edition, McGraw-Hill, New York, 1987.

[31] Ruiz, J. M.: The Basic Theory of Power Series Vieweg-Verlag, Braunschweig, (1993).

[32] V. D. Waerden: Algebra I, Springer-Verlag, Berlin, Heidelberg, New York, (1993)

[33] Whitney, H.: Analytic extensions of differentiable functions defined on closed sets Trans. Am. Math. Soc. 36, 63-89 (1934).

[34] YosidA, K.: Functional Analysis. Springer, Berlin, 1980. 